\title{
Innovations in ex vivo Light Sheet Fluorescence Microscopy
}

\author{
Pablo Delgado-Rodriguez ${ }^{\mathrm{a}}$, Claire Jordan Brooks ${ }^{\mathrm{a}}$, Juan José Vaquero ${ }^{\mathrm{a}, \mathrm{b}}$, \\ Arrate Muñoz-Barrutia a, b, * \\ a Departamento de Bioingeniería e Ingeniería Aeroespacial, Universidad Carlos III de Madrid, Madrid, Spain \\ ${ }^{\mathrm{b}}$ Instituto de Investigación Sanitaria Gregorio Marañón, Madrid, Spain
}

\section{A R T I C L E I N F O}

\section{Article history:}

Received 12 March 2021

Accepted 12 July 2021

Available online 19 July 2021

\section{Keywords:}

Microscopy

Fluorescence

Architecture

Image analysis

Quantification

Multimodal

\begin{abstract}
A B S T R A C T
Light Sheet Fluorescence Microscopy (LSFM) has revolutionized how optical imaging of biological specimens can be performed as this technique allows to produce 3D fluorescence images of entire samples with a high spatiotemporal resolution. In this manuscript, we aim to provide readers with an overview of the field of LSFM on ex vivo samples. Recent advances in LSFM architectures have made the technique widely accessible and have improved its acquisition speed and resolution, among other features. These developments are strongly supported by quantitative analysis of the huge image volumes produced thanks to the boost in computational capacities, the advent of Deep Learning techniques, and by the combination of LSFM with other imaging modalities. Namely, LSFM allows for the characterization of biological structures, disease manifestations and drug effectivity studies. This information can ultimately serve to develop novel diagnostic procedures, treatments and even to model the organs physiology in healthy and pathological conditions.
\end{abstract}

๑) 2021 The Authors. Published by Elsevier Ltd. This is an open access article under the CC BY-NC-ND license (http://creativecommons.org/licenses/by-nc-nd/4.0/).

\section{Motivation}

Light Sheet Fluorescence Microscopy (LSFM) (Huisken et al., 2004) is a microscopy technique that has become widely popular over the past few years as a way of providing 3D reconstructions of fluorescent-labeled biological samples or specimens (Girkin and Carvalho, 2018; Keller and Dodt, 2012; Lim et al., 2014; Mano et al., 2018; Albert-Smet et al., 2019). The images are obtained from the fluorescent signal emitted by the sample after light excitation. Its main advantages over other microscopy methods are 3D imaging capabilities, high spatial and temporal resolution and low phototoxicity. Ex vivo samples, such as histological sections and whole animal organs, can be efficiently imaged using this method after a fluorescent labelling process and a clearing protocol to render them transparent. Some of these clearing procedures are toxic, which must be considered for their correct application. LSFM presents considerable advantages for the analysis of ex vivo samples compared to other approaches such as the conventional optical microscopy (Fricker, 1993) used for histopathology, which only provides two-dimensional information and requires to physically

\footnotetext{
* Corresponding author. Escuela Politécnica Superior, Universidad Carlos III de Madrid, Avda. Universidad, 30, 28911, Leganés. Madrid, Spain.

E-mail address: mamunozb@ing.uc3m.es (A. Muñoz-Barrutia).
}

cut the samples to obtain thin layers. Another widespread microscopy modality, confocal microscopy (Pawley, 2006), can be used to obtain 2D slices from a specific inner plane of a specimen, using a confocal pinhole to eliminate out-of-focus fluorescence signal and image a single point of the plane. This process does not require sample slicing; however it is not well suited for imaging many planes of the same sample due to the long acquisition times required. In contrast, a single LSFM application on a sample provides an overall 3D reconstruction. This method is significantly faster than confocal, which allows for rapid volumetric data acquisition and can also be applied for live image of adequately transparent specimens.

From a medical perspective, ex vivo LSFM imaging provides fundamental 3D knowledge on tissue structure to allow for a better understanding of damage caused by diseases. 3D images can be much more informative than the selection of $2 \mathrm{D}$ views obtained by a confocal microscope. With LSFM it is possible to observe detailed, complete architectures 3-dimensionally. Fluorescent labeling is used to locate key structures, which can be used, for example, to map disease related features or drug distribution inside an organ. Despite the many advantages of this technique, one of the main drawbacks is the size limitation on the sample. In classical LSFM architectures the sample size is restricted by the light emitter from the side and the receiving lens on top of it. This arrangement of 
perpendicular objectives makes the system more size restrictive than other modalities such as bright field or confocal microscopy, which do not have lateral objectives interfering in the sample's placing. Another aspect to consider when deciding to use LSFM is the need for transparency, which restricts its use to ex vivo cleared samples, as it is currently impossible to use LSFM for in vivo screening, except with naturally transparent specimens like zebrafish embryos.

Many experimental works using LSFM to image ex vivo samples present visual qualitative outcomes from the visualization of structures by human experts (Dodt et al., 2007; Jährling et al., 2008). Others compare their LSFM images to different imaging modalities, such as light microscopy to demonstrate that the fluorescence method can be used as an alternative to classical histology procedures (Lloyd-Lewis et al., 2016; Glaser et al., 2017). In recent years, however, there have been several innovations to those initial LSFM applications. These innovations come in the form of novel microscope architectures allowing for faster acquisition (Yang et al., 2015) or larger sample accommodation (Reder et al., 2019), among others. There have also been efforts to improve the state of quantification-oriented image analysis of LSFM, which aims to obtain mesoscopic features from the images using objective and indepth image analysis techniques. This type of image processing includes automatic segmentation techniques using manually segmented ground truths, for targeting structures like blood vessel networks (Hu et al., 2017a) or detecting cells and other small bodies (Frasconi et al., 2014). This automatization can result in significant time saving and reduce the required human labor while providing more objective and reproducible results. These and similar analyses show how LSFM can be used to obtain objective measurements that may help in tasks such as observing the spatial response of a certain organ to a treatment, quantifying differences between samples or in some cases creating mathematical models of biological systems to predict their behavior (d'Esposito et al., 2018). Additionally, multimodality imaging that combines LSFM with other types of medical images like Optical Projection Tomography (OPT) (Mayer et al., 2014) or confocal microscopy (Köster and Haas, 2015) can provide more information than either technique alone, using the additional image as a structural complement.

In this manuscript, we offer a general frame for the use of LSFM on ex vivo samples and a relation of the newly available methods to work with it. We first review the fundamentals and story of LSFM, including the first examples of its use for ex vivo biological samples and a series of classical architectures. Then we examine the usual methods for ex vivo imaging of cleared samples, including sampletypes, architectures, staining and clearing techniques. The next section of the article is focused on recent projects expanding LSFM capabilities, which take into account novel technological advances, quantitative image processing techniques and multimodal combination of LSFM with other imaging modalities. Finally, we offer a conclusion on how these approaches can open the way for emerging new applications on the biomedical field.

\section{Light sheet microscopy fundamentals}

Light-Sheet Fluorescence Microscopy refers to a set of techniques which acquire images by sweeping a tissue sample using a thin layer of light that excites fluorescent molecules present in that plane and causes them to send a fluorescent signal to be detected by a lens, usually at $90^{\circ}$ of the light sheet, focused on the plane of interest. 2D Images obtained are specific to the fluorescence emitted in that single plane, excluding any signal coming from the rest of the sample and allowing visualization of inner structures without the need of any physical sectioning of the sample. This technique avoids any unnecessary fluorescence excitation of the surrounding planes and thus reduces phototoxicity and premature fluorophore degradation compared to other methods such as wide field fluorescence microscopy. Many LSFM applications require fluorescent labelling, either through genetic modifications or antibody binding, tagging certain structures so that they emit a light signal to be detected by the lens. The labeled areas will be shown as fluorescent signal in the images, highlighting specific features in the sample. Autofluorescence (no labeling) can also be used to get an overall structural reconstruction relying on inherent fluorescence of the tissues without focusing on specific targets. During an LSFM imaging session, it is necessary either to use a transparent specimen (such as zebrafish) or to clear the sample in order to modify its refractivity index and allow light to traverse through it without distortion.

The earliest biological application of LSFM technology corresponds to A. H. Voie et al. (1993) (Voie et al., 1993), with a threedimensional analysis of cleared guinea pig cochlea using an LSFM system termed Orthogonal-plane Fluorescence Optical Sectioning (OPFOS). The impact of the method was low until several years later it was used for bacteria imaging in oceanography (Fuchs et al., 2002), this time under the name of Thin Light-Sheet Microscopy (TLSM). Eventually, LSFM gained attention from the biological community after it was successfully used to image living zebrafish embryos using a similar setup (Huisken et al., 2004), which in this case was referred as Selective Plane Illumination Microscopy (SPIM), the name this original architecture would be known for during the next years.

\subsection{Classical architectures}

The oldest and more widespread versions of LSFM architecture consist of variations on the SPIM model, the original and most basic system. This setup features single plane lateral illumination of the sample and $90^{\circ}$ between the fluorescence plane and the lens (Fig. 1A). The original system used by J. Huisken et al. (2004) offered a lateral resolution below $6 \mu \mathrm{m}$ and a penetration depth of around $500 \mu \mathrm{m}$. Several variations on the same basis have been implemented by using different lenses, laser beams or applying either single plane or double plane illumination, with two beams directed towards the sample from opposite directions. Double plane illumination appears in the Ultramicroscope architecture (Becker et al., 2008; Dodt et al., 2007) and allows for a more uniform light-sheet, which is useful on larger samples given that the light is attenuated as it travels through the tissue (Fig. 1.B). Double plane illumination partially avoids shadows that particles can produce when illuminated from only one side. These types of microscopes rely on stationary light sheets, which facilitates the construction of custommade devices following these principles.

Different setups rely on moving illumination to improve image quality. Multidirectional SPIM (mSPIM) (Huisken and Stainier, 2007) is an alternative to the SPIM architecture that includes a moving light sheet to illuminate the specimen from several angles (Fig. 1C), reducing shadowing that appears in more static systems. Other alternatives include DSLM (Digital Scanned Light Sheet Microscope) (Keller et al., 2008), where a scanned light sheet is used instead of a constant one. It works by moving a linear laser signal horizontally through the image to create the planes and vertically to change from one to another. The procedure increases light efficiency and allows all lines on each plane of the specimen to be illuminated with the same intensity, among other benefits (Fig. 1D). As a development on the DLSM setup, the Confocal Light Sheet Microscope (CLSM) (Silvestri et al., 2012) also uses a thin line of light which is swept along a specimen's horizontal plane (confocallike illumination), but includes the addition of a confocal detection system by means of a slit that allows to detect fluorescence coming 

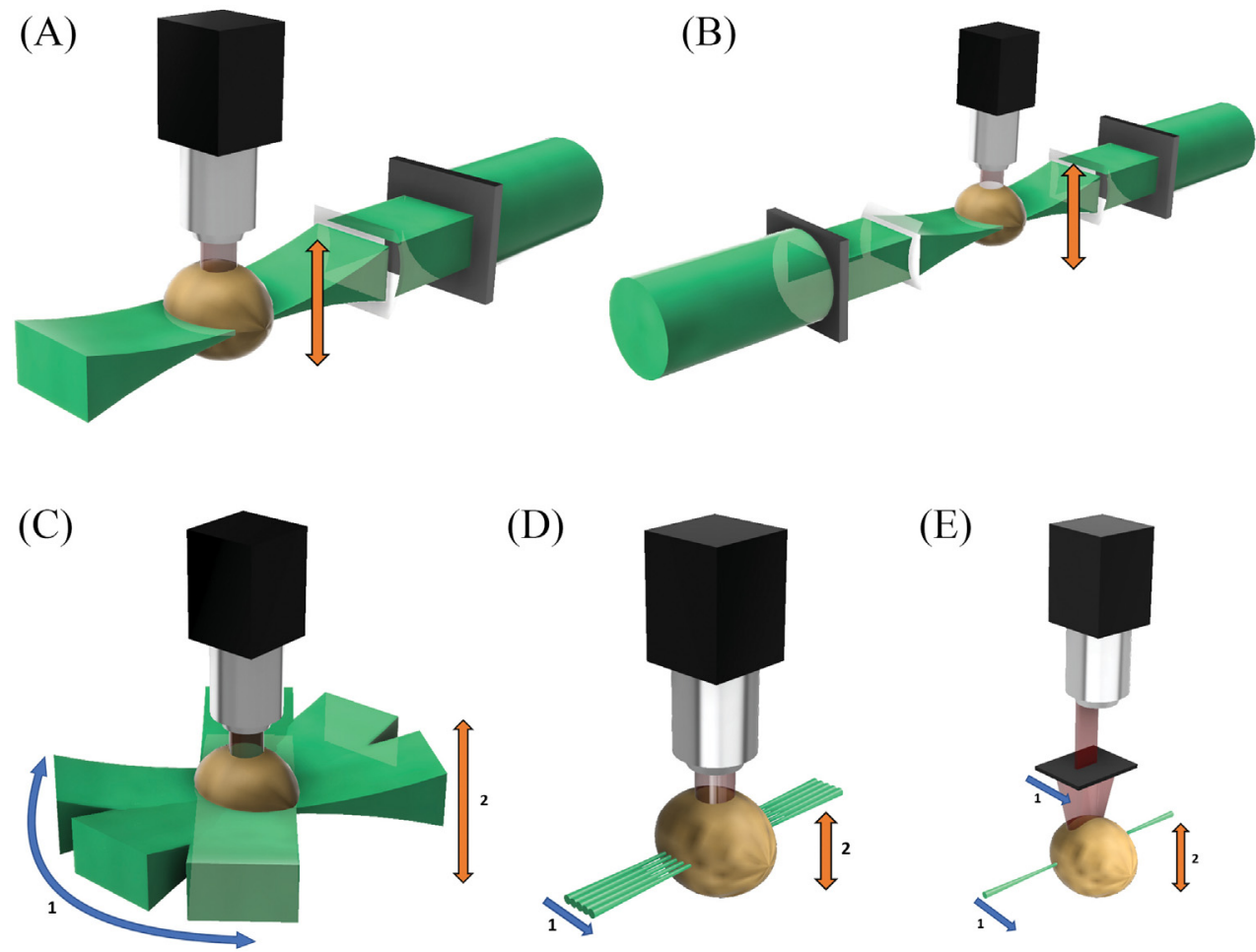

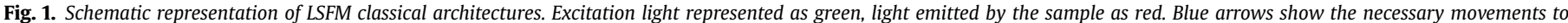

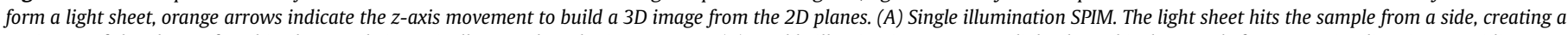

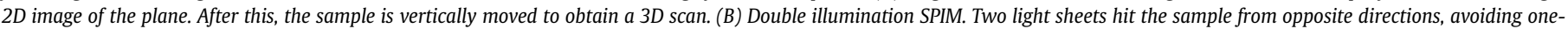

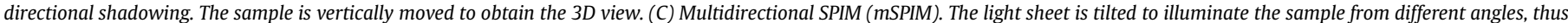

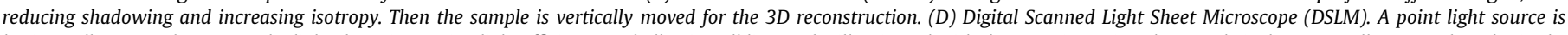

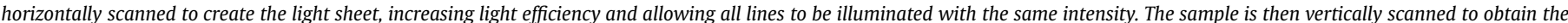

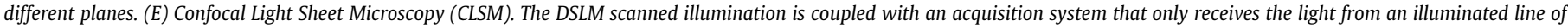

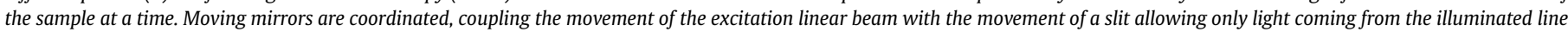

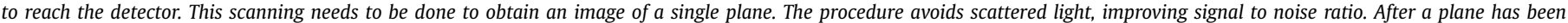
completed, vertical movement of the sample and horizontal scanning of the illumination beam for each plane provides the volumetric image.

only from the excited line at each moment. Both emitter and detector are coordinated to focus on the same line, creating a plane with each sweeping sequence by means of these excitation lines. The procedure prevents scattered light from being imaged, increasing the Signal to Noise Ratio (SNR) since the out of focus light is avoided, but requires precise coordination of emitter and detector (Fig. 1E).

While many research groups create their own systems, in recent years some commercial models have also been released, becoming widespread as an alternative to avoid the time spent and the complications derived from building a custom microscope. For instance, LaVision BioTec Ultramicroscope (LaVision BioTec, Bielefeld, Germany) (Hu et al., 2017a; Klingberg et al., 2017; Zundler et al., 2017; Thierbach et al., 2018; Pan et al., 2016; Gómez-Gaviro et al., 2017; Hägerling et al., 2017; Noë et al., 2018; Rocha et al., 2019) or Lightsheet Z.1. (Carl Zeiss AG, Oberkochen, Germany) (Isaacson et al., 2017, 2018; Saritas et al., 2018) are popular among the community. These and other commercial alternatives have allowed easy access to LSFM for research groups who would not have considered the possibility otherwise, creating more opportunities for innovations on the technique.

\subsection{Ex vivo applications of Light Sheet Fluorescence Microscopy}

Given that LSFM requires transparency of the observed volume, biomedical use of this technique primarily relies on ex vivo imaging of a cleared organ or histological section. This type of study can offer preliminary knowledge as a basis for further work on disease treatment. Several studies have shown the possibilities of ex vivo LSFM. The work by Dodt et al. (2007) constitutes a clear reference for the field, presenting successful images of both cleared Drosophila Melanogaster and mouse samples. Posterior research has proven how this modality can be used on different types of ex vivo samples with promising results, like 3D reconstruction of human skin samples (Abadie et al., 2018), as shown in Fig. 2, where several details of the skin internal structure can be observed. This section presents an overview of the most common clearing techniques, sample types and labeling procedures used in LSFM studies.

\subsection{Clearing techniques}

To adequately perform a light sheet study and obtain accurate information from the interior of the sample, the chosen clearing technique is crucial. Since the popularization of LSFM among the biomedical community, a variety of clearing techniques to render the samples adequately transparent has been developed (Ueda et al., 2020a). Of these techniques, Benzyl Alcohol/Benzyl Benzoate (BABB) is one of the oldest and most widely used. This method was developed from a mixture designed by Walter Spalteholtz (Spalteholtz, 1911), which constituted the first application of techniques for rendering samples transparent. BABB application consists of dehydrating the tissue and submerging it in the reagent, substituting the extracted water to render it transparent. It has been used in many occasions on ex vivo samples (d'Esposito et al., 
(A)
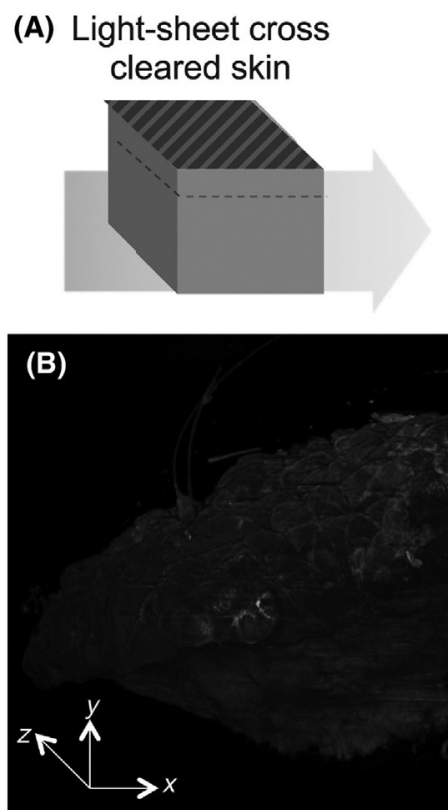

(D)
Optical section

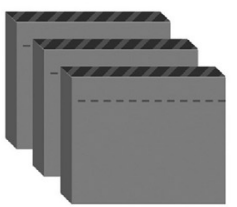

3D volume reconstruction

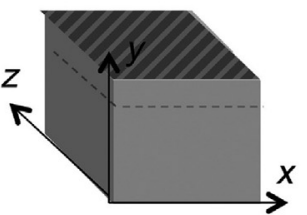

(C)
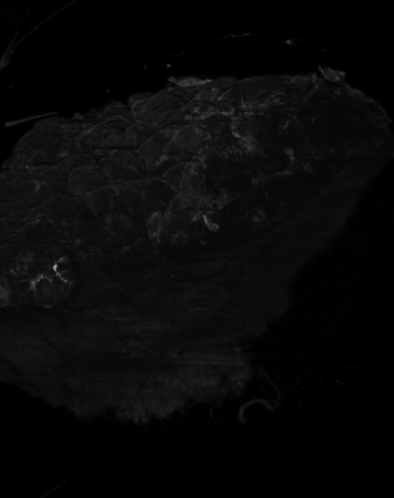

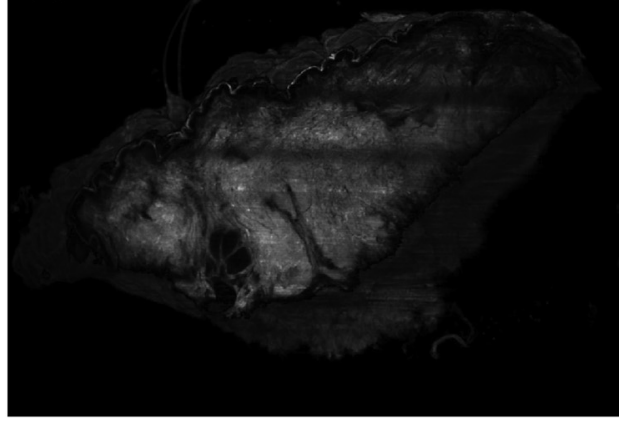

(E)

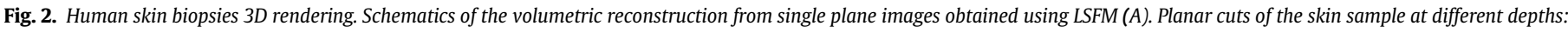

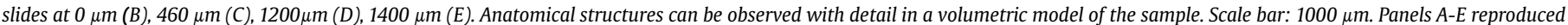
from Abadie et al. (2018).

2018; Dodt et al., 2007; Frasconi et al., 2014; Fuchs et al., 2002; Hu et al., 2017a; Huisken and Stainier, 2007; Jährling et al., 2008; Keller et al., 2008; Köster and Haas, 2015; Mayer et al., 2014; Reder et al., 2019; Voie et al., 1993; Yang et al., 2015). Alternatively, a series of techniques based on the method 3DISCO (3D imaging of solventcleared organs) (Ertürk et al., 2014) have been successfully applied by other groups. 3DISCO uses BABB but applies two other solutions to the sample before it: Tetrahydrofuran (THF) for dehydration and dibenzyl ether (DBE) as a clearing reagent that preserves fluorescence better than BABB. A variation of the method, uDISCO (ultimate DISCO) (Pan et al., 2016) focuses on increasing fluorescence duration (Zhang et al., 2019). Another variety, iDISCO (immunolabeling-enabled three-dimensional imaging of solventcleared organs) (Renier et al., 2014), is based on 3DISCO to create a robust, fast and inexpensive clearing method (Hu et al., 2017b; Tanaka et al., 2017). As an alternative, iDISCO+ is a modified technique that reduces shrinkage (Rocha et al., 2019; Renier et al., 2016; Perin et al., 2019). An alternative to the previous 3DISCO techniques is CLARITY (Saritas et al., 2018; Chung et al., 2013), originally an acronym of Clear Lipid-exchanged Acrylamide-hybridized Rigid Imaging/Immunostaining/In situ hybridization-compatible Tissuehydrogel: a technique providing a hydrogel scaffold to maintain the tissue structure after clearing, yielding better structural images than BABB. As a drawback, this procedure is less reproducible and scalable due to the need of a hydrogel, which adds complication to the protocol. Variations of CLARITY have been developed by different research groups to suit their needs. For example, the Passive Clarity Technique (PACT) (Yang et al., 2014) is applied as a way of clearing whole organs in a fast manner without the need of external forces (Isaacson et al., 2017, 2018; Stefaniuk et al., 2016). To keep a high-performance clearing but at the same time reduce the need of specific devices for the hydrogel (as happened with CLARITY), the CUBIC procedure, which stands for Clear, Unobstructed Brain/body Imaging Cocktails (Susaki et al., 2015), was created. This technique focuses on reproducibility and scalability and consists of two steps: hydrophilic solvents immersion to remove lipids, and perfusion with a second reagent to match refractive index. Fig. 3 shows different clearing techniques applied to human skin biopsies for illustration of a clearing procedure's effects, showing how tissue transparency is greatly increased after clearing using any of the methods. More in depth reviews on clearing procedures are common in the literature (Ueda et al., 2020b; Gómez-Gaviro et al., 2020). In this section we presented an overview of some of the most relevant methods.

\subsection{Sample types}

From a biomedical perspective, the analysis of human samples is the most direct way of obtaining relevant information to be later used in a clinical scenario. Light sheet microscopy is a powerful tool 

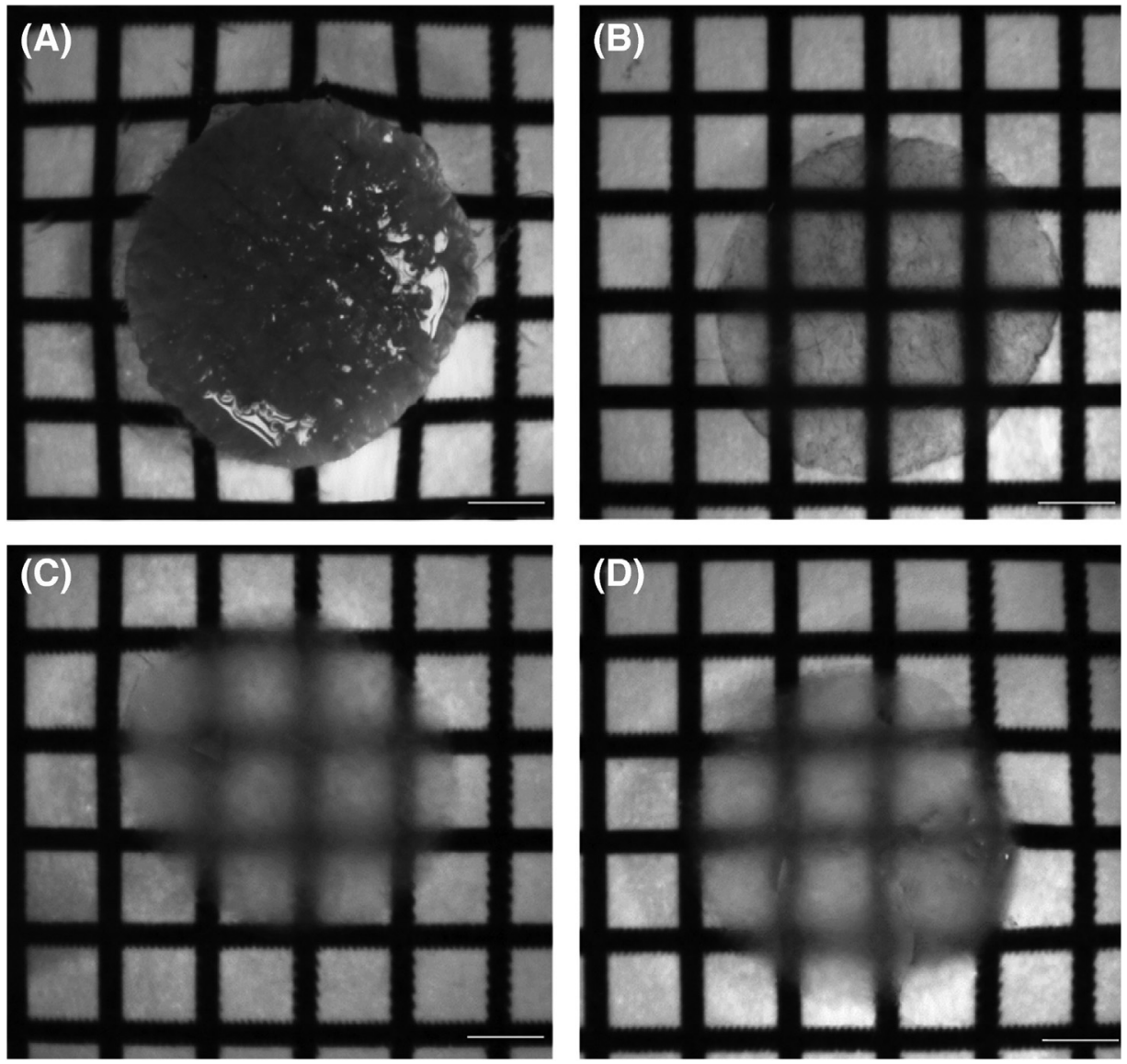

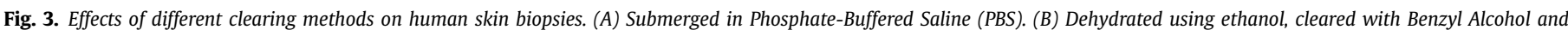

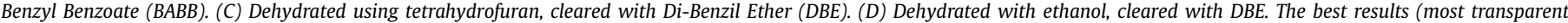

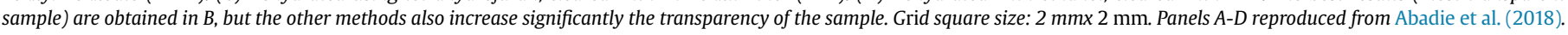

to characterize several abnormal behaviors in the organism, such as malignant tumoral structures. This has been achieved for bladder tumors by identifying lymphatic vessel structural alterations to characterize them (Tanaka et al., 2018), and for colorectal carcinoma samples by studying lymph nodes to determine the differences in cell shape between pathological and nonpathological samples (Nojima et al., 2017). Other tumoral samples include pancreatic tissue to study the behavior of cancer cells, determining that venal invasion is almost universal in pancreatic cancer, which explains its high lethality (Hong et al., 2020). LSFM images have been used as well as a comparable and faster alternative to traditional H\&E biopsies in order to look for cancer markers in samples like prostate (Glaser et al., 2017; Reder et al., 2019) and breast (Glaser et al., 2017; Chen et al., 2019) tissue.

Other analysis of human samples focus on skin to characterize the structural effects of diseases like lymphedema (inflammation of the lymphatic vessels) (Hägerling et al., 2017) or epidermic hyperplasia (Abadie et al., 2018), using it to visualize in 3D the enlargement on the epidermal layer on skin samples with images comparable to the ones obtained from 2D sample sections by conventional light microscopy (Fig. 4). Light sheet microscopy has also been applied to different organ samples aiming to demonstrate its viability on several tissues such as lung, coronary arteries or brain arteries (Nojima et al., 2017). Imaging of human brain samples has also been proven successful, even as a validation for in vivo Magnetic Resonance Imaging (MRI) studies (Morawski et al., 2018).

Despite being the most relevant type of specimen from the medical perspective, human samples are not always available. In these cases, animal samples provide valuable insight as a mean of in-depth exploration of biological structures or disease effects that can serve as a basis for medical development. Some of the most widely studied animal ex vivo samples have been the brain and parts of the neural system, especially in mice. In multiple occasions, mouse brains have served to characterize the results of certain clearing and/or imaging protocols (Silvestri et al., 2012; GómezGaviro et al., 2017; Pan et al., 2016), but they have also been analyzed using immunolabeling to obtain a clear visualization of neural tissue, allowing to localize neural cells (Frasconi et al., 2014). Combining this procedure with other labeling allows to obtain combined neural/vessel information (Di Giovanna et al., 2018; Hama et al., 2011). A different type of labelling, using lectin, has been chosen as a common procedure to observe the vessel tree structure of these brain samples (Jährling et al., 2009), relying on segmentation techniques to get exact representations of this network (Hu et al., 2017a, 2017b; Di Giovanna et al., 2018). Besides, Formalin-induced fluorescence labelling was able to identify different structures of the mouse brain by negative cell staining (Leischner et al., 2010). Not only the brain, but mice spinal cord has also been studied using LSFM to observe neural regeneration (Ertürk et al., 2012), obtaining information on neural recovery to wounds, with the aim to help repair this tissue in pathological cases.

Besides mouse models, brains from other animals normally appear as study subjects using Light sheet microscopy. An example of this is rat brains, which have served as examples of large nervous tissue samples that can be adequately resolved by LSFM (Stefaniuk 

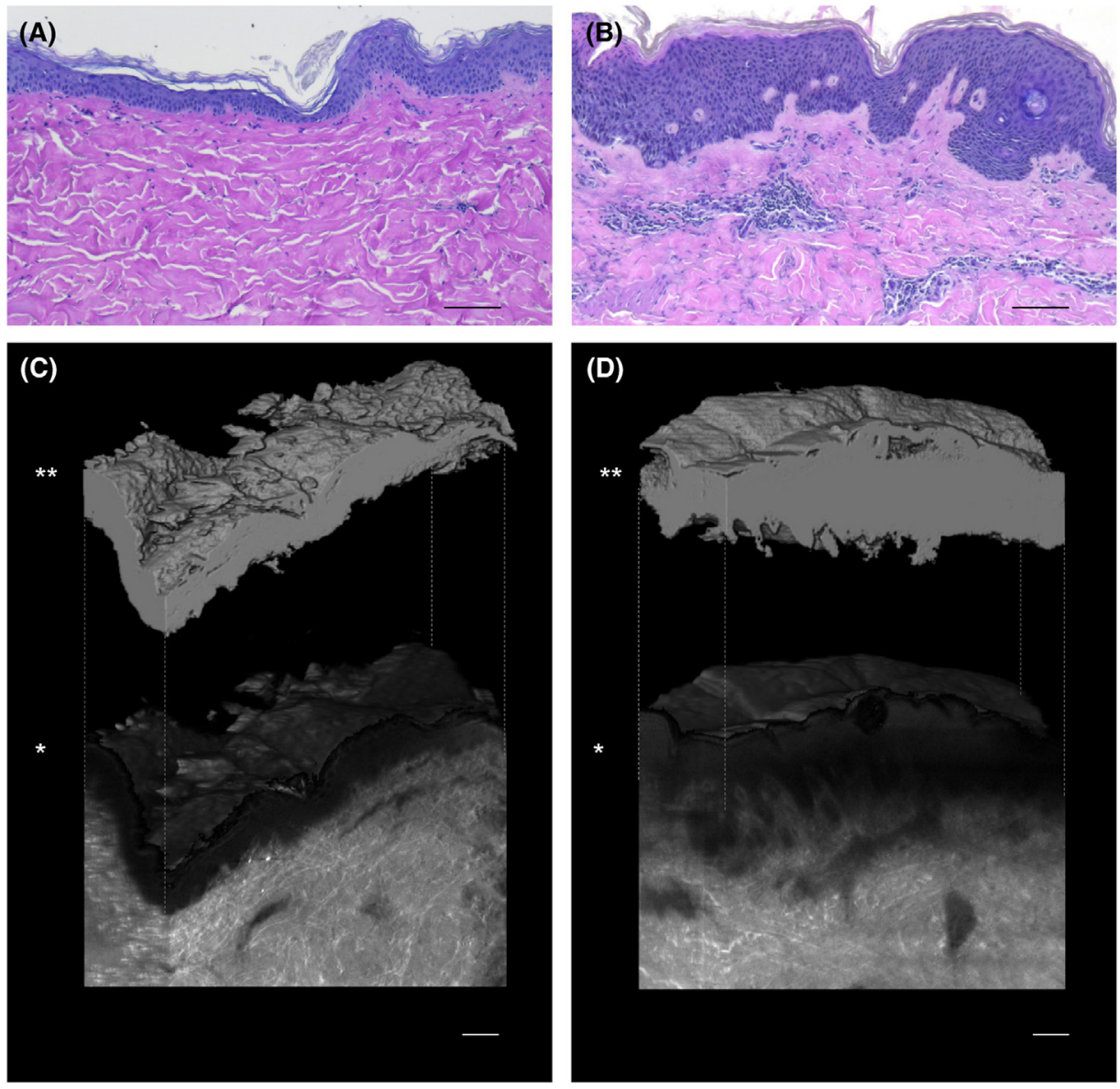

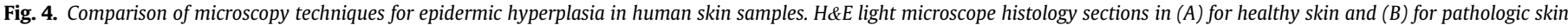

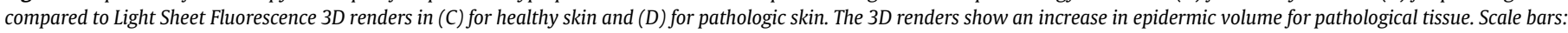
$100 \mu \mathrm{m}$. Panels A-D reproduced from Abadie et al. (2018).

et al., 2016), but also songbird brain in order to obtain more information about the process of song learning and production for these animals, with the aim to find a basis for the study of the homologous region in humans. The High Vocal Center (HVC), the brain area where these functions are localized, was visualized by fluorescent labelling using Adeno Associated Viruses (AAV) (Rocha et al., 2019).

LSFM potential has been also tested on different types of animal organs other than neural tissue. These include mice hearts, obtaining, for example, a 3D view of the lectin labeled heart vascular tree (Nehrhoff et al., 2017). This can serve as a preliminary step to more exhaustive evaluation of vessel remodeling after injury. Other organs such as mice kidneys have also been studied to determine the evolution of the glomeruli count when affected by some disease (Klingberg et al., 2017). Renal pathologies reduce the normal activity of this organ, which is usually translated in a lower number of active filtering units (glomeruli).

Not only organs, but also entire animal bodies have been used to test Light sheet microscopy's capacity to extract highly detailed images from large samples. One of the classical specimens to test LSFM is a whole Drosophila Melanogaster (Pan et al., 2016; Järling et al., 2010). Nevertheless, complete mouse bodies can also offer useful insights of their anatomy and physiology, labelling molecules specific to a certain disease, for example calcium labelling to study the effects of Duchenne muscular dystrophy on a body (Bozycki et al., 2018). These whole-body studies are evolving to more complex analyses as modelling and quantification techniques become more relevant. That is the case for C. Pan et al. (2019), who have recently studied the behavior of human cancerous cells as they spread through the mouse body, locating the different micrometastases and the therapeutic particles sent to them. Such studies provide detailed views of the morphology on an entire biological specimen, targeting specific areas or obtaining an overall view through autofluorescence. This way, the relationship of certain body regions with each disease's development can be analyzed.

\section{Exploring further LSFM possibilities}

LSFM is a powerful technique which is evolving as a greater number of research groups become aware of its potential as a powerful tool to obtain 3D images of specifically labeled particles. The first approaches to this modality relied on devices that were not trivial to operate and offered acceptable but improvable results in terms of resolution. Clarification techniques needed to be tested, and many research projects provided results that were given in a qualitative form, assessing the correct functioning of a specific "architecture plus clarification method" combination. In recent years there have been many advances in LSFM, including new microscope architectures, multimodality combinations with other imaging techniques, and new image analysis methods that had not been previously applied to LSFM images. Novel approaches like the ones presented in the following section provide the tools to extract additional information to characterize previously unexplored diseases or increase our knowledge on already studied ones. Some of 
these methods may become the basis for the development of new treatments or diagnosis procedures that were not previously possible.

\subsection{Novel architectures}

In recent years there have been many advances in LSFM in the form of new microscope architectures and multimodality combinations with other imaging techniques, broadening the horizons of this microscopy technique.

As LSFM becomes popular between a wider range of researchers, its limitations and possible improvements are being highlighted. This has resulted in a series of alternative architectures that can help mitigate some of the principal drawbacks of LSFM, from facilitating sample placing to increasing resolution, which allows for image analysis techniques to work with more accurate information. Over the last years a series of novel technological advances for LSFM have been proposed, developed from the configurations mentioned in Section 2. These advances consist of new microscope architecture designs that improve different features of the imaging procedure, including:

\subsubsection{Speed improvement}

Acquisition time is an important concern when using LSFM to obtain images of large samples. It facilitates the operator work and results in a larger number of imaged samples per unit of time, which can be useful to provide a higher amount of information for an experiment. To reduce acquisition time by increasing the imaging speed, Dual-slit confocal light sheet microscopy (Yang et al., 2015) was proposed as an evolution of the CLSM (Silvestri et al., 2012) concept. Dual-slit CLSM uses two scanning linear beams, operating in opposite directions, each of them coordinated with a different slit to receive the excitation signal (Fig. 5A). Thus, each half of the image is obtained from one of the scanning beams, increasing the rate of acquisition. A similar technique is Confocal multiview light-sheet microscopy (Medeiros et al., 2015), which also combines double illumination with confocal slit detection to reduce scattering. Each side is illuminated with a sweeping line of light moving to the opposite direction of each other to allow two moving slits to obtain their signals at the same time. Images are weighted by a sigmoidal function and combined. The process allows for double illumination more quickly than conventional microscopes. Aiming for a faster microscope that preserves resolution, the SPED Light Sheet Microscope (Tomer et al., 2015) features an increase in the detector lens depth of field by means of a transparent piece in front of it. This way, it is not necessary to move the detector together with the light-sheet. Instead, the light sheet can be moved on its own, providing it remains in the focus of the detector. This allows for an increase in speed acquisition since it is dependent of the galvanometer operation speed, much faster than the camera acquisition rate. All the setup results in a high-quality volumetric acquisition system which speed is only limited by the cMOS camera acquisition rate, which could be improved in future years. Compared to more classical architectures, which speed is limited by the piezo motors moving the sample, the SPED system can acquire several thousands of volumes in a second. The device has been used to image entire zebrafish brains, demonstrating that the method is applicable to whole organs.

\subsubsection{Light sheet refinement}

The properties of the light sheet can greatly influence the output of an LSFM study. Light sheet uniformity is important to obtain isotropic images independent from the illumination direction. Samples illuminated from a single side show a series of artifacts due to shadowing, which reduces the Signal to Noise Ratio (SNR). This can distort the representation of certain sections of the images, presenting an inaccurate result that can lead to less reliable biomedical conclusions. To deal with these issues, methods like Multidirectional digital scanned light-sheet microscopy (Glaser et al., 2018) (mDSLM) have been developed. mDSLM is based on mSPIM (Huisken and Stainier, 2007) and DLSM (Keller et al., 2008) in an effort to illuminate the sample from as many angles as possible and reduce shadowing artifacts. The mDSLM architecture relies on an elliptical gaussian light beam with different numerical apertures for the $\mathrm{x}$ and $\mathrm{y}$ directions (parallel and orthogonal to the light sheet). The elongated size of the beam in the $\mathrm{y}$ direction provides angular diversity, instead of relying on mechanical movement of a linear beam (as in mSPIM). This beam is horizontally swept through the sample to construct a virtual light sheet (as in DSLM). The schematics of this process appear in Fig. 5.B. The results of mDSLM against SPIM, MSPIM and DSLM have been evaluated on eosin labeled human breast images, where adipose tissue and stroma could be observed (Glaser et al., 2018). mDSLM showed a decrease in shadowing compared to SPIM and DSLM, while it also exhibited a contrast improvement when evaluated against SPIM and mSPIM. These two characteristics allowed for a more precise imaging of the alveolar structures of the breast tissue, whose borders could be determined better than with the previous methods.

Besides uniformity, the sheet's thickness can be a determining factor. A thinner light sheet provides higher resolution in the z-axis, exciting only a very small section of the sample and thus reducing out of focus light. It makes 3D reconstructions in general smoother and more accurate, which again results in more reliable outcomes of image analysis. To approximate a perfectly thin and constant layer an LSFM architecture has been developed based on dividing the observation field into tiles (Gao, 2015), each of them illuminated by a small light sheet. This setup creates multiple regions of thin light sheet, which decreases the overall thickness through the sample. All these tiles are then combined to form the complete image. Another approach to reduce the light sheet's thickness is the Axially Swept Light Sheet Microscopy (ASLM) (Dean et al., 2015), which moves the light sheet generated by a Gaussian beam in a synchronized manner with a slit detector, such that the section imaged at each moment is the one illuminated by the thinnest part (waist) of the light sheet (Fig. 5C). This setup is able to offer a Z-axis resolution of $390 \mathrm{~nm}$ throughout a $216 \mu \mathrm{m}$ x $162 \mu \mathrm{m}$ x $100 \mu \mathrm{m}$ field of view, significantly better than the one provided by gaussian (conventional) light sheet, which is only able to achieve similar axial resolution for a field of view of $\sim 2 \mu \mathrm{m} \times 2 \mu \mathrm{m}$ and becomes degraded as the region increases. Building on this idea, cleared Tissue ASLM (ctASLM), a modification on the previous system, was presented as an optimization of the concept for cleared ex vivo samples (Chakraborty et al., 2019). As a different approach that does not focus on illumination from different angles, another recent technique called Tilted light sheet microscopy with 3D point spread functions (TILT3D) (Gustavsson et al., 2018) relies on modifying the 3D PSF (Point Spread Function) (Von Diezmann et al., 2017) that determines the transformation suffered by the light when received by the detector. By combining a tilted illumination and a long axial range PSF, it becomes possible to image single particles using high NA (Numerical Aperture) objectives very near the plate.

\subsubsection{Different illumination directions}

The difficulty of a conventional SPIM arrangement to accommodate large samples has led to the development of systems featuring illumination and detection paths below the samples, at a $45^{\circ}$ angle of the horizontal while maintaining the $90^{\circ}$ angle between both lenses. This allows for whole organ imaging without any spatial restraints on the specimen since it is placed on an open 
(A)

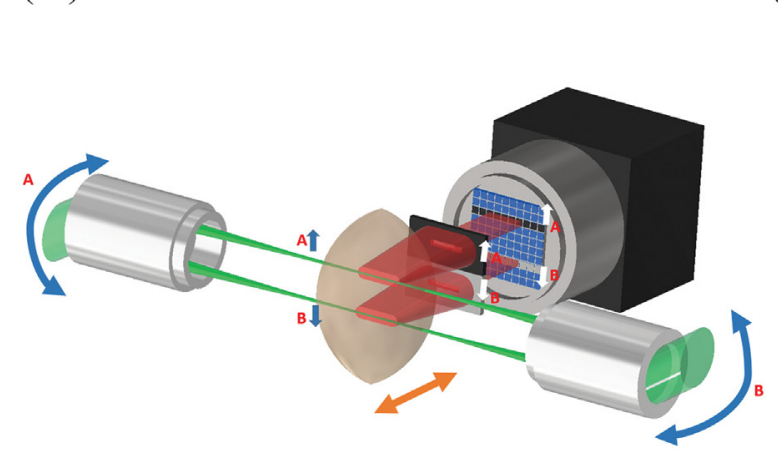

(B)

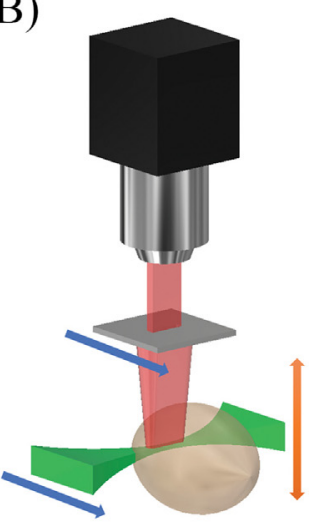

(C)

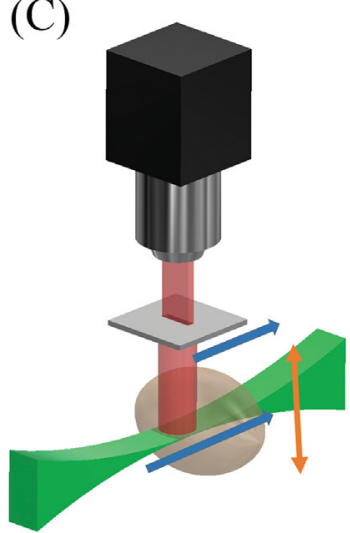

(D)

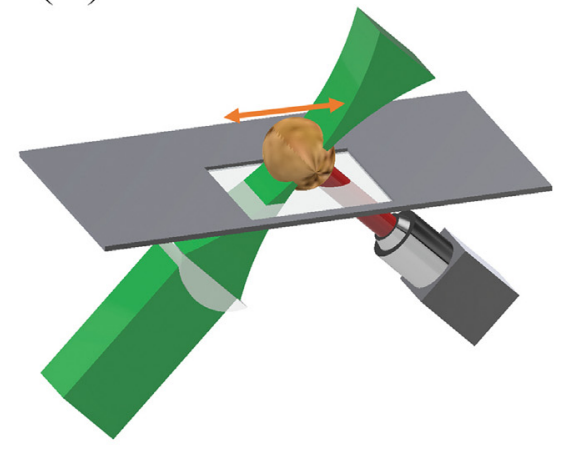

(E)

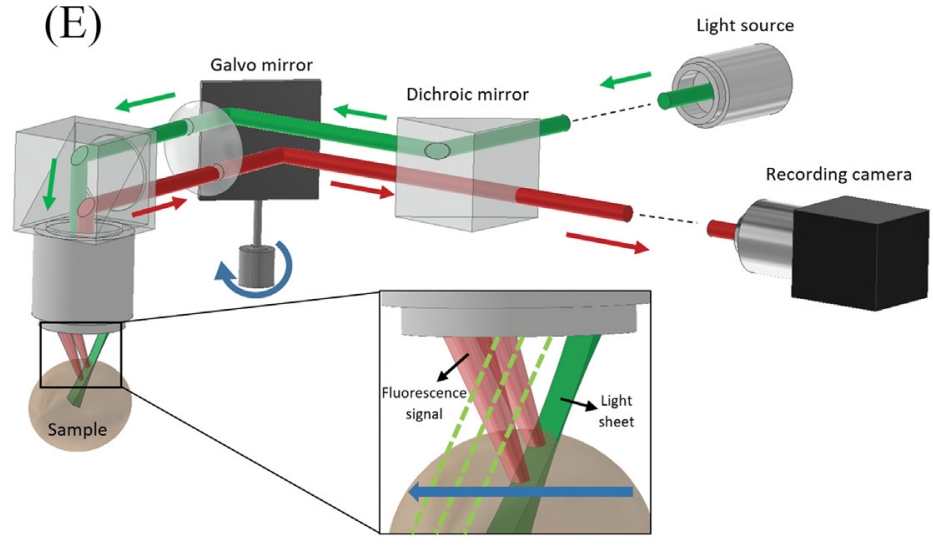

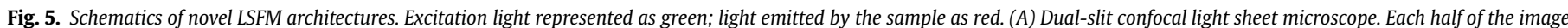

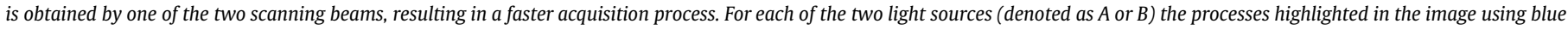

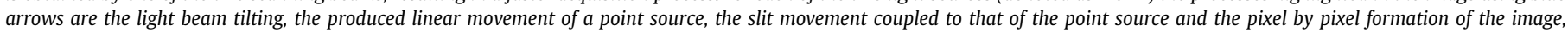

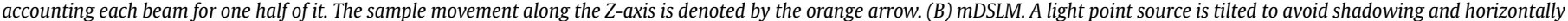

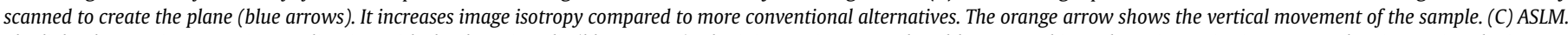

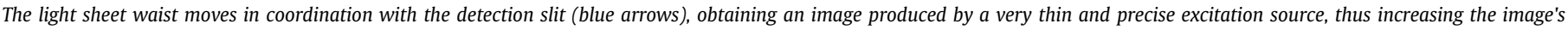

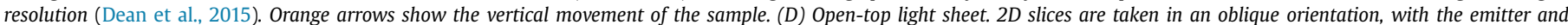

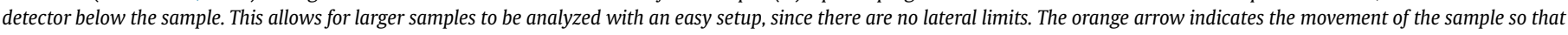

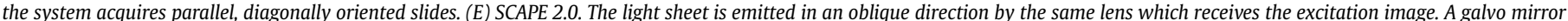

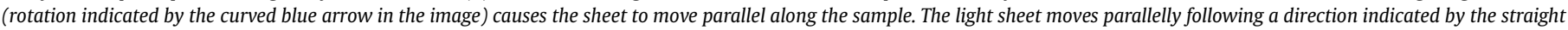

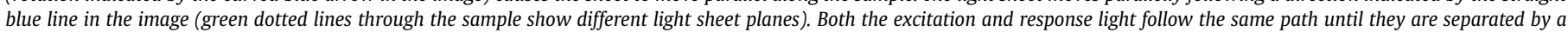

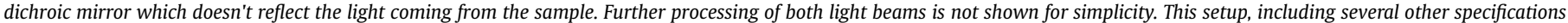
explained in Voleti et al., 2019 . maintains a high resolution and greatly increases the acquisition speed compared to relying on motors to move the sample.

plate. Large samples can be easily placed on the surface without any lateral constraint, as in more classical devices. This broadens the sample range that can be imaged using LSFM in order to obtain new results from larger samples. It allows for example for easy mounting of centimeter-sized human samples (Reder et al., 2019; Glaser et al., 2017; Chen et al., 2019). Too thick samples still cannot be adequately imaged due to the limited penetration depth of the light sheet in any architecture.

In this type of architecture with $45^{\circ}$ illumination angle from the bottom, slices are acquired in an oblique manner, which requires a more complex reconstruction process than horizontal slicing (Reder et al., 2019; Glaser et al., 2017; Chen et al., 2019; Strnad et al., 2016). A summary of the system is seen in Fig. 5D. The convenient sample placing also makes the mounting process faster, which increases the processing speed of histopathology samples, such as prostate core biopsies (Reder et al., 2019) or breast lumpectomy margins (Chen et al., 2019) (of around $1 \mathrm{~cm} \times 1 \mathrm{~cm} \mathrm{x} 0.5 \mathrm{~cm}$ ). A similar system, Diagonally Scanned Light-Sheet Microscopy (Dean et al., 2016), instead uses a CLSM-based architecture, and applies an oblique slicing for faster imaging of thin cells.

Other innovative setups include Single objective SPIM (SoSPIM)
(Zagato et al., 2017), which consists of the use of microfabricated sample holders featuring small mirrors in the intersections between their walls and bottom. These micromirrors allow to create a system with its laser beam coupled with the receiving lens, so that a vertical laser signal is reflected to create the horizontal plane, which is then swept through the sample. This arrangement is useful for incorporating LSFM with conventional optical microscopes without the need for cumbersome lateral structures, thus making this technique more accessible for laboratories which cannot afford more expensive commercial LSFM microscopes. As an alternative to this, a similar architecture was developed using an Atomic Force Microscopy (AFM) cantilever coated with an aluminum layer to reflect a vertical laser beam on top of the sample to a horizontal plane (Gebhardt et al., 2013). This setup allows to couple the light emission and reception in a single objective, as the SoSPIM does.

Similar imaging architectures include Light sheet theta microscopy (Migliori et al., 2018), which places both detector and emitters in the same side of the sample, avoiding lateral size restrictions without compromising speed or resolution. Two light sheet emitters are arranged symmetrically at both sides of the receiving lens, roughly at a $60^{\circ}$ angle from it and around $30^{\circ}$ to the horizontal 
plane. The receiving lens is placed between the light sheet emitters and includes a moving slit system which, coordinated with both emitters, acquires a signal only from the thin line of the specimen illuminated by both light sheet's waists at the same time. This arrangement allows for high-resolution fast imaging of very large samples (such as whole mouse brains), including the interior regions of the samples. The acquisition speed is higher than more classical architectures. Another alternative is Swept ConfocallyAligned Planar Excitation (SCAPE) (Bouchard et al., 2015), a system in which the excitation light sheet is emitted in an oblique angle from the same lens that receives back the fluorescent signal. The excitation light sheet is continuously swept back and forth and along the sample by means of a polygonal oscillating mirror, keeping the scanned detection plane stationary. This setup offers a faster acquisition than conventional systems, which are limited by sample movement. The speed improvement can be of the order of 240 times faster for SCAPE. This architecture's features provide an adequate frame for live sample imaging, including high depth of focus to observe large specimens. A new version of the SCAPE architecture was developed recently, referred to as SCAPE 2.0 (Voleti et al., 2019). It works on the same oblique illumination principle, using the same lens to emit the light sheet and receive the fluorescent signal, however the polygonal mirror has been replaced for a more standard system of galvo (tunable) mirrors that in turn allow for a similar functionality in a more robust manner. The architecture of this microscope is depicted in Fig. 5E.

\subsubsection{Other innovations}

Apart from the previously presented areas, other relevant proposals offer interesting possibilities to the scientific community. One of them is a hyperspectral light sheet microscope that illuminates the sample by means of a scanned light sheet and then descans the signal emitted by it so that the different wavelengths can be analyzed separately at the same time (Jahr et al., 2015). This way, the signals offered by different fluorophores can be obtained from a single application, reducing the difficulties to combine separate images and the time spent in the process, which can serve to simultaneously monitor either several drugs or drug diffusion and morphology changes. In addition, a series of projects have been released during the years which aim to provide an open-source framework for the construction and operation of standard LSFM systems. This helps the community of LSFM users to grow, as more research groups are able to build their own system with smaller effort and cost. These initiatives include OpenSPIM (Pitrone et al., 2013), an open controlling software for different LSFM varieties, as well as OPT (Sharpe et al., 2002). As a recent alternative for centimeter-size cleared samples, the mesoSPIM initiative (Voigt et al., 2019) provides detailed instructions to assemble an open source hardware ASLM system. This structure, combined with the corresponding open source software, results in a specialized tool for the analysis of cleared samples, compared to many of the architectures found in the literature which focused more on real-time acquisition capabilities for studies such as embryo development. A large field of view in the microscope increases the region's size, while anisotropy is countered by the ASLM technology. In general, mesoSPIM provides an easy access for everybody to study cleared samples with optimal results.

\subsection{Quantitative analyses of mesoscopic features}

Quantifiable data obtained from analyzing LSFM images and measuring mesoscopic features can provide valuable knowledge. There are many applications to this type of data acquisition, including cell characterization; for instance, a distribution of ovarian follicle diameter obtained by manual segmentation (Lin et al., 2017) can offer insight on the development of premature ovarian failure (POF) and polycystic ovary syndrome (PCOS). Structural segmentation also provides relevant data and can serve as a framework for further modelling. For instance, a segmentation of the lung bronchial tree obtained with autofluorescence can be used as a scenario for nanoparticle tracking to test for a certain treatment effectiveness (Yang et al., 2019). Obtaining such measurable data can benefit the researchers by providing extra levels of information, but manually extracting these features is time and labor intensive. As an alternative, several automatic processing techniques have been recently implemented, reducing the previous requirements, increasing reproducibility and allowing for faster acquisition of a larger amount of data. The following sections present a summary of automatic methods for extracting specific characteristics from LSFM images. These analyses aim to provide researchers with reliable biological information to understand the body's functioning.

Before an image analysis method is applied, specific considerations on LSFM images need to be taken. The most crucial of these is image size. LSFM is able to produce a large number of highresolution planar images. For large samples, the whole specimen does usually not fit inside the field of view of the microscope. The most common workaround to this is to obtain several image tiles of a manageable size that will be later stitched together to form the entire specimen. The size of this block representation of the image can be in the terabyte order. This tile representation of the images needs to be correctly handled for visualization and further processing, so the registration process that aligns and combines all tiles together to form the whole image is an important piece of the workflow. A number of tools have been recently developed to deal with LSFM acquired images, including the KLB lossless compression format developed at the Keller Lab (Janelia Research Campus), able to reduce the data size 30-500 fold (Amat et al., 2015). Specific tools include Fiji plugins like BigDataViewer (Pietzsch et al., 2015), able to manage and visualize this kind of large microscopy files, and BigDataProcessor2 (Tischer et al., 2020) to process large images. Also dedicated stitcher algorithms like MosaicExplorerJ (Tosi et al., 2020) help overcome the problems of reconstructing such large images. Recently, the LOBSTER (Tosi et al., 1093) package for MAT$\mathrm{LAB}$ was published as well. It consists on a set of several image processing algorithms specifically tuned for fluorescence images, with an emphasis on adequately processing large samples such as the ones usually analyzed by LSFM.

Quantitative methods can be classified according to the type of information which is being measured. The next sections show the most common applications.

\subsubsection{Structural microscopy segmentation}

Image segmentation consists of delimiting certain regions of the image, which allows for the collection of measurements from a whole structure, organ, or feature of interest. Using a system to automatically segment these structures is a useful tool, but the algorithm performance needs to be evaluated against manual segmentations to check for reliability. Some of the applications of automatic segmentation methods include the analysis of blood vessel networks, which allows to measure parameters related to its shape and distribution, providing additional information about blood system function under different conditions. This type of segmentation has been conducted on mouse cerebral vessels using a convolutional neural network (CNN) (Hu et al., 2017a) trained with manually segmented patches where an expert highlighted the pixels corresponding to mouse brain vessels. These manual segmentations were used to train the $\mathrm{CNN}$, a complex nonlinear function that, once tuned, was able to infer for each pixel of the new images a probability of belonging to a blood vessel. Alternative 
algorithms involving Markov random fields (Di Giovanna et al., 2018) or fuzzy local information C-Means clustering (FLICM) (Hu et al., 2017b) have resulted in similar automatic segmentation of this same structure. Despite blood vessels are usually the structure of interest, other relevant sections have also been segmented. The cardiac trabecular network, for instance, shows the inner structure of the heart walls and can be extracted very precisely from LSFM images using a machine learning approach such as subspace approximation with augmented kernels (Saak) (Ding et al., 2020). Different structures can be obtained in detail from LSFM volumes, be it axonal projections of neurons in the mouse brain (using methods like Fully Convolutional Neural Networks, FCNNs) (Syenina et al., 2020) or disease-related structures. Image processing software tools like IMARIS (from Oxford Instruments) allow for surface segmentation, which can help characterize structures like fungal infection development patterns in murine lung for different models of immunosuppression (Amich et al., 2020). Another one of these tools, AMIRA (a product of ThermoFisher Scientific), offers functionalities like a semi-automatic filament tracking algorithm that can serve to segment filaments after the user specifies a starting and ending point. This has its uses in tasks like retinal blood vessel network analysis, allowing also to measure features like vessel lengths, volumes, branching points or Euler numbers (Chang et al., 2021).

Image segmentation can be instrumental for derived applications. One of them is the creation of a brain atlas that works well with LSFM images. This has been done by automatic registration of brain region segmentations coming from the atlas "Common Coordinate Framework version 3 developed by the Allen's Institute of Brain Science" (AIBS CCFv3, obtained from other microscopy modalities) with a model brain created from LSFM images (Perens et al., 2020). Another utility that can be developed from the results of automatic image segmentation algorithms are mathematical models to mimic physiological functions, thus allowing to understand better the working principles of the body. Light Sheet microscopy has been used to obtain a segmentation and skeletonization of the circulatory system in a mouse fat pad tissue sample (Kennel et al., 2020). The skeletonization allowed to construct a graph model showing the interconnections between vessels and to identify several sub-volumes of closely connected vessels. These sub-volumes were also analyzed from a functional point of view, taking into account perfusion modelling to obtain a representation of the functional organization of tissue. A similar technique to LSFM, Optical Projection Tomography (OPT), has also been used to obtain 3D segmentations of fluorescently labeled, cleared mouse tumors, building a mathematical model for drug diffusion upon them (d'Esposito et al., 2018). This modality could as well be substituted by LSFM to perform similar analyses that can help predict the behavior of micro or nanoparticles introduced in a body.

All these procedures provide ways of overcoming one of LSFM main problems: the data management issues due to a large volume of $2 \mathrm{D}$ images obtained in a single study. Manually identifying the structures of interest of a whole image can be challenging when the amount of information is so large, and 3D segmentation is not a trivial task, many times requiring the expert to go through each individual 2D slide in order to achieve the most reliable results possible. Using automatic methods reduces human workload, increases speed and offers reproducibility: a single algorithm will usually offer more consistent results than several different human experts, each of them analyzing the image from their own viewpoint.

\subsubsection{Particle localization}

Besides the segmentation of organs or large biological structures, automatic procedures are also applied for finding and characterizing particles such as cells in the imaged organs or samples, using again manually annotated pictures as a ground truth. This method can be used for a variety of purposes, such as to track certain drugs inside the body, to observe the behavior of a cell population, or to quantify the changes in shape of small structures due to some disease. In cell culture studies, the localization of individual cells is important to analyze their evolution over time, while in ex vivo samples specific cells that have been immunolabeled can be observed within the whole sample. The localization of these small structures can provide information on cell densities in different parts of the body.

The applicability of these analyses on LSFM data has been demonstrated, for example, to localize mouse brain cell centroids using a mean shift clustering algorithm (Frasconi et al., 2014) or Purkinje cells in a mouse cerebellum (Silvestri et al., 2015) which can be later grouped by means of a k-means clustering method, providing clear boundaries that show the cell distribution inside this organ in a more exact manner, as can be observed in Fig. 6A. This way, it was possible to create a map of all Purkinje cells and visualize their structure. Studies like this one can yield significant knowledge on some pathologies. For instance, the localization of gaps inside the cerebellum cell organization is believed to be related to autism disorders. Similar analyses have been performed on different samples to locate targets like c-Fos positive neurons under different stress situations in the mouse brain (Renier et al., 2016). LSFM has also been used to observe cells from samples other than neural, like murine fetal tissue (Scherzinger et al., 2016). To obtain accurate cell nuclei segmentation in this type of specimen a random forest was trained to classify each pixel as cell or background using the Ilastik toolkit (Sommer et al., 2011) to separate cells from the background. After this, a rainfall simulation was applied to the clusters to find intensity peaks. The final process was to use a watershed algorithm to separate the cells in each cluster. Another method that has been applied for cell segmentation on human brain samples features a Mixed-scale Dense Network (MSD) (Thierbach et al., 2018). With an analogous approach, mouse kidney glomeruli have also been observed, segmented and quantified, allowing for shape observation and glomeruli density calculations (Klingberg et al., 2017). In Fig. 6B the clear contrast between these glomeruli and the background can be appreciated. All these methods allow for precise localization and shape measurements to observe the effect of a disease on individual cells.

Particle localization can be applied not only to find cells, but also for tasks like analyzing pancreatic islets to detect pharmacologically induced changes in B cells during diabetes using a U-net (Roostalu et al., 2020), or assessing tumor spreading. This last application is shown in the recently presented study by C. Pan et al. (2019), where a Light sheet microscopy is used to obtain 3D images of a whole mouse body which has been injected with human tumoral cells, immunolabeled to find the tumor cells and cleared to achieve transparency. By means of a fully convolutional network inspired in the U-net architecture (Ronneberger et al., 2015) small micrometastases can be found and quantified at the cellular level, together with the distribution of antibody-coupled drugs applied to the mouse. A schematic of a U-net-like network can be seen is illustrated in Fig. 7. The segmentation provided by the CNN allows for an estimation of drug efficacy based on the proportion of drug molecules which were able to bound a micrometastasis. For application, the neural network obtains maximum intensity projections of the image along each of the three dimensions, evaluates the projections separately as $2 \mathrm{D}$ images, building three respective probability maps of each pixel being tumor and combines these three maps to rebuild a 3D probability volume. A segmentation mask is then obtained from the volume and used to get a precise distribution of both tumors and drugs at the cell level, thus helping 


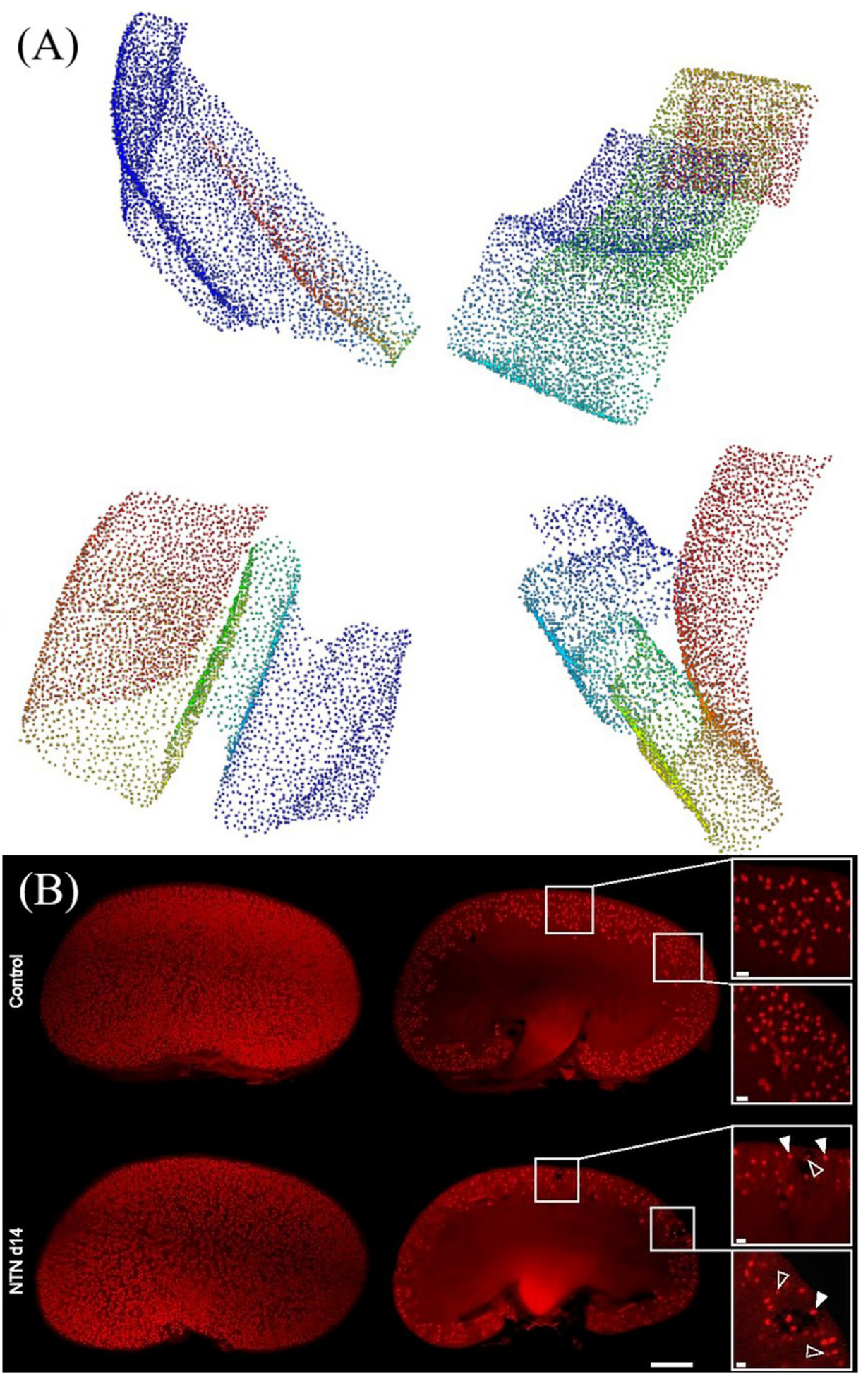

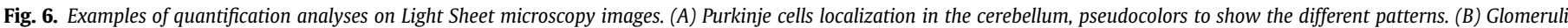

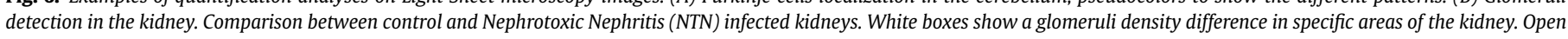

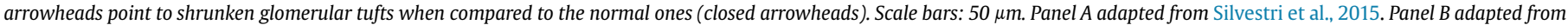
Klingberg et al., 2017.

to understand the dynamics of cancer spreading and treatment to a deeper level.

In the deep learning field, alternative approaches are being considered to bypass some limitations like the difficulty to access a large number of LSFM labeled data. To this end, the use of
Generative Adversarial Networks (GANs) is becoming popular. This type of algorithms allows to generate synthetic data and consist on a pair of neural networks: one of them discriminates real from fake images while the other generates synthetic images to try and fool the discriminator into believing they are real. This has been used, 


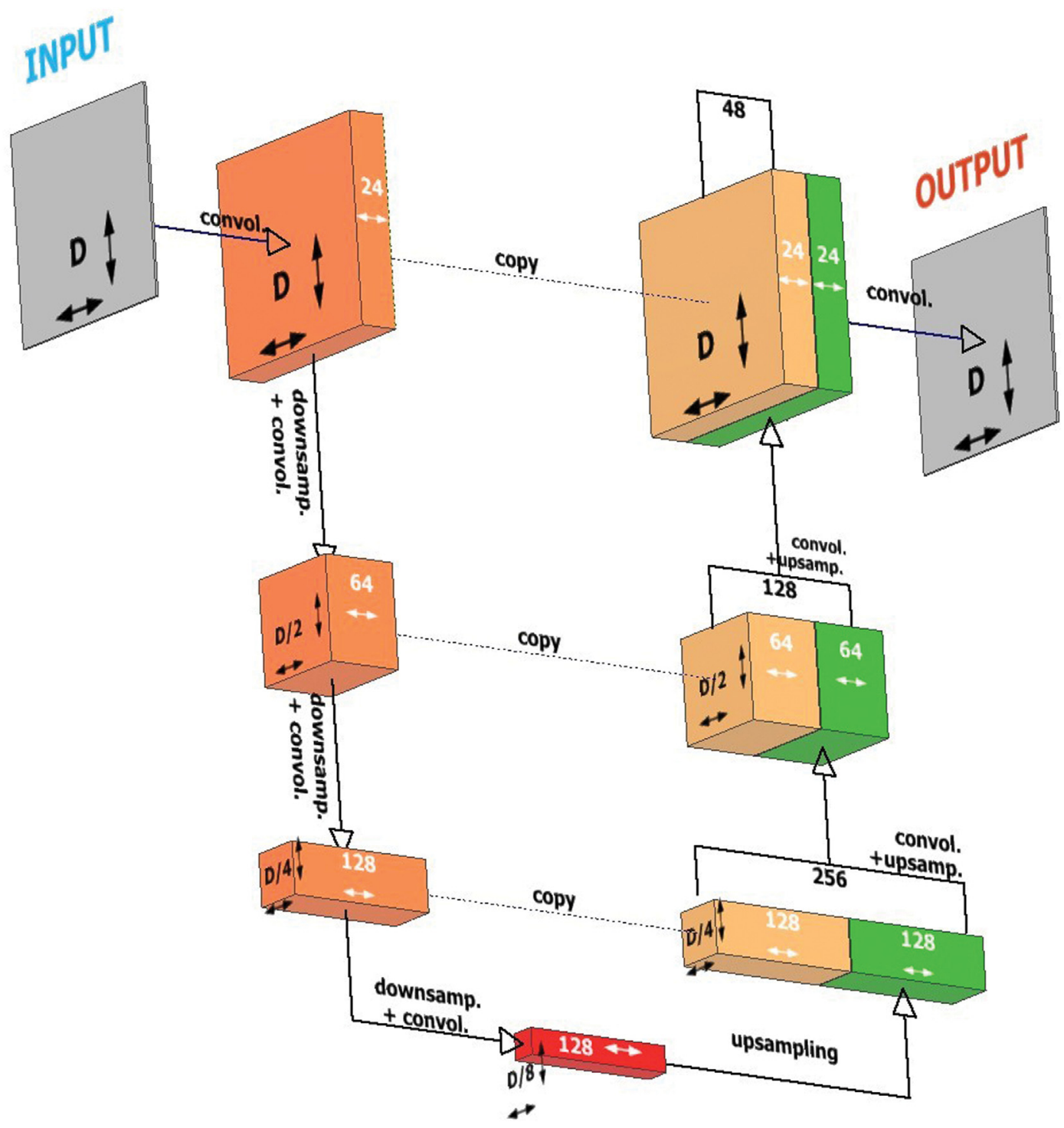

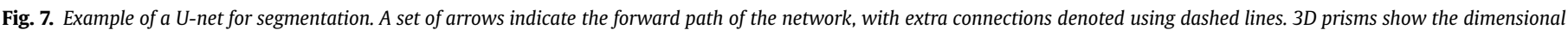

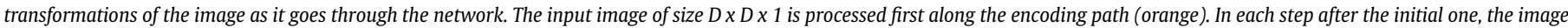

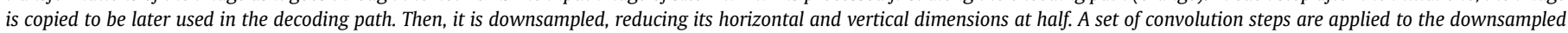

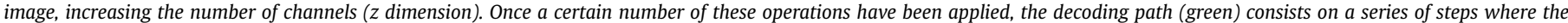

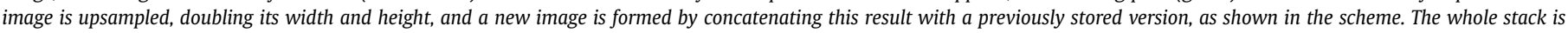

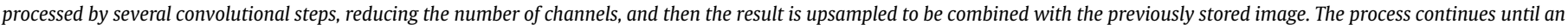

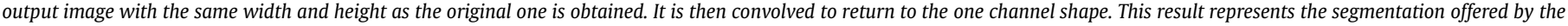
net.

for instance, to transfer the knowledge of a network trained with confocal microscopy images to another net able to analyze LSFM images (Guo et al., 2019), using the more prevalent confocal images as a basis for a particle segmentation algorithm. Projects like this greatly expand the possibilities provided by previous microscopy methods to analyze the body in an exact manner.

\subsubsection{Alternative data representations}

Segmentation methods can be combined with data transformations that allow additional information to be extracted from LSFM data. For example, blood vessel skeletonization refers to encoding a segmented vessel structure as the system of nodes and vertices connecting them. This representation is better suited for some calculations like vessel tortuosity, heterogeneity or ramification, as well as to form a basis for mathematical models. One way of obtaining such representation involves thresholding LSFM images to obtain a blood vessel segmentation, which is then automatically skeletonized to get a clearer model of its structure. Once the skeletal scheme is obtained, several parameters can be measured, like vessel radius. This can be done, for instance, by centering a sphere in each of the points of the vessel skeleton and expanding it until a certain proportion of it is outside the vessel (Kennel et al., 2018b). Some other applications of the skeletonization process include urinary tissue vessel segmentation (Tanaka et al., 2017) or lymphatic vessels analysis (Tanaka et al., 2018). Mathematical models explaining blood flow can also be created with this technique, as seen in P. Kennel et al. (2018a) LSFM images can also serve as a base for simulations, as shown by A. Hann et al. (Hahn et al., 2020), who used the microvessel structures observed in healthy and tumor-bearing mouse brains by light sheet 
microscopy and applied a mathematical model to them, obtaining the corresponding change in Nuclear Magnetic Resonance (NMR) signal for that region. This representation accounts for the one that would be produced in a specific voxel of a full Magnetic Resonance Imaging (MRI) study. By training a Support Vector Machine (SVM) algorithm with a series of those simulations it was possible to build a classifier that allows to identify cancerous voxels in MRI, which can help diagnose this disease in a faster and more accurate way.

Other transformations can be applied to the obtained segmentations to yield more convenient representations. For instance, LSFM data from a mouse intestine have been transformed by rearranging the $3 \mathrm{D}$ volume obtained in such a way each intestine layer is unfolded to form a different image, based on a certain separation distance between layers defined by the user (Candeo et al., 2016). Villi are also segmented to obtain specific measurements like villi density. This transformation can help study intestinal disease models from a more convenient point of view. The information provided by this and the previous methods can shed some light on the local effects of diseases for biological tissues from a different perspective.

\subsection{Multimodal imaging featuring LSFM}

LSFM is a potent technique, but its possibilities can be expanded through a combination with other imaging modalities. This combination can be obtained by a coupled acquisition using two image modalities at the same time so that their results are aligned, and both can be merged into a single overall result. It is also possible to acquire each modality separately and later register the images so that their results can be combined. This allows for the fluorescent labeled sections of LSFM to be more precisely localized inside a body by the additional information provided by the other modality.

A useful technique to complement LSFM is Optical Projection Tomography (OPT) (Sharpe et al., 2002), which also requires a cleared sample to reduce scattering. Similar to a standard CT procedure, OPT takes several views of the sample at different angles and uses a reconstruction algorithm to create a $3 \mathrm{D}$ visualization. Its main advantage over LSFM is it can retrieve nonfluorescent signal in addition to fluorescent signal (albeit at a lower resolution than LSFM). The combination of this technique with SPIM has been demonstrated using a variety of setups. One such combination, OPTi SPIM, includes an illumination source which is placed below the sample for the OPT analysis, and a laser light sheet directed through it at a $90^{\circ}$ angle for fluorescent excitation (SPIM) (Mayer et al., 2014). The detector is placed on top of the sample and receives either one of the two signals, be it OPT structural information or SPIM fluorescently labeled areas. For OPT acquisition, the sample is rotated such that several images can be acquired and later reconstructed, while for SPIM it is moved along one axis, storing 2D slice images that are later combined as a 3D. Despite the different procedures, both three dimensional images are already registered, since the sample is placed in the same spot, which is one of the main advantages of this combination of methods. The OPT signal provides a structural framework which helps to contextualize the location of the SPIM-detected labeled molecules.

As another example of multimodal imaging, Leica Microsystems has developed a confocal laser scanning microscope with SPIM system (Leica SP8 DLS) (Köster and Haas, 2015). This setup allows for easily switching between confocal and light sheet modes, introducing new possible analyses. Using a vertical light sheet that travels upwards to the sample and is then reflected horizontally using two mirrors, a dual sided light sheet is generated through the sample. Both modalities can also be combined; for example, confocal microscopy may be used to activate photoconvertible proteins, that will be later imaged using the LSFM module. In addition, the combined system has the advantage of reducing costs as it integrates two devices into a single one.

\section{Discussion and future directions}

LSFM is an emergent technique for the analysis of ex vivo cleared samples, yielding important information that help to characterize organ structure and particle distribution. Ex vivo imaging of cleared samples provides insight on tissue functioning for a variety of human and animal tissues, which allow for a deeper understanding of disease behavior. Fluorescent labeling of key structures, together with a suitable clearing technique, are crucial to obtaining the desired information using LSFM technologies.

Novel approaches to the LSFM technique have been explored in recent years. In respect to the technological advances, changes in architecture have led to improvements in several fields such as the improved acquisition speed of the SPED system. Some setups like in SCAPE (Bouchard et al., 2015) and SCAPE 2.0 (Voleti et al., 2019) have adapted sample mounting architectures which allow for the imaging of large samples. We have also seen improvement in illumination uniformity from systems such as ASLM (Dean et al., 2015), which keeps the light sheet's waist at the detection area at all times. From a software perspective, several open source platforms for image acquisition have been developed, such as openSPIM (Pitrone et al., 2013) and mesoSPIM (Voigt et al., 2019), which have reduced the barrier of entry for researchers to use LSFM technology.

It is possible to yield additional information by utilizing LSFM in combination with imaging modalities such as OPT, to provide general spatial information for the fluorescently labeled particles (Mayer et al., 2014), or with Confocal microscopy, which reduces photobleaching compared to confocal on its own.

There have also been developments in the image processing techniques available for LSFM data which are increasing the ability to identify and quantify mesoscopic features from the mesoscopic images. Several research groups have applied advanced techniques to automatically segment structures, detect cells and particles, obtaining their distributions, or measuring features such as vessel diameter or tortuosity. With these techniques, it is possible to use LSFM images with fluorescently tagged specific particles to obtain conclusions on many aspects, like drug concentration or structural changes after a disease or treatment. The amount of information present in these large size images can be analyzed in a fast and accurate manner using these techniques.

Image processing developments in LSFM, particularly in automatic feature extraction methods, greatly reduce the time and labor requirements of analyzing LSFM images, constituting a promising field that will likely be explored in depth in the following years. A particularly interesting field where these methods can be useful is the development of mathematical models from ex vivo LSFM images to explain organ functions and disease development. The detailed 3D framework observed in these images provides the possibility of analyzing and modelling a wide range of structures and their interactions. Future studies could develop this at a deeper level to build in silico organ models where new treatments can be preliminarily tested without the need of real animals or patients. These quantitative analyses can be complemented by newly available microscope architectures that provide extra capabilities, offering images with higher resolution and general quality along with less complicated sample preparation and image acquisition. This would result in more precise models with a more reliable behavior. What is more, thanks to several open source initiatives, LSFM is becoming increasingly accessible for researchers of different disciplines, opening the doors to new ideas on possible applications and features to be analyzed, novel modality combinations with other biological signals and new setup modifications to adapt this diversified need. 
Despite all the advances on the field, there are still some limitations to the usage of LSFM for ex vivo samples. Sample clearing remains in general an unavoidable step that adds a certain complication to the procedure and can be a source of errors. Choosing the adequate clearing method is thus a very determining step. High resolution images of large samples require huge volumes to be stored, which, combined with the still long acquisition times makes it difficult to acquire samples from animals bigger than mice or rats. As new light sheet microscopes are developed during the next years, these size limitations will probably be overcome, providing the means to study the whole organism function and structure.

As a conclusion, the new advances in LSFM imaging for ex vivo samples offer very promising results that can serve as a foundation for future research with this modality and continue to develop the technique to a higher level. Despite certain limitations, this is a promising field that can give rise to relevant results in the biomedical field in the future years.

\section{Funding}

This work was produced with the support of the Spanish Ministry of Science, Innovation and Universities (TEC2016-78052-R, RTC-2017-6600-1, PID2019-109820RB-100, FPU19/02854).

\section{Declaration of competing interest}

The authors declare that they have no known competing financial interests or personal relationships that could have appeared to influence the work reported in this paper.

\section{References}

Abadie, S., Jardet, C., Colombelli, J., et al., 2018. 3D imaging of cleared human skin biopsies using light-sheet microscopy: a new way to visualize in-depth skin structure. Skin Res. Technol. 24, 294-303.

Albert-Smet, I., Marcos-Vidal, A., Vaquero, J.J., et al., 2019. Applications of light-sheet microscopy in microdevices. Front. Neuroanat. 13, 1-15.

Amat, F., Höckendorf, B., Wan, Y., et al., 2015. Efficient processing and analysis of large-scale light-sheet microscopy data. Nat. Protoc. 10, 1679-1696.

Amich, J., Mokhtari, Z., Strobel, M., et al., 2020. Three-dimensional light sheet fluorescence microscopy of lungs to dissect local host immune-Aspergillus fumigatus interactions, 11, 1-18.

Becker, K., Jährling, N., Kramer, E.R., et al., 2008. Ultramicroscopy: 3D reconstruction of large microscopical specimens. J. Biophot. 1, 36-42.

Bouchard, M.B., Voleti, V., Mendes, C.S., et al., 2015. Swept confocally-aligned planar excitation (SCAPE) microscopy for high-speed volumetric imaging of behaving organisms. Nat. Photonics 9, 113-119.

Bozycki, L., Łukasiewicz, K., Matryba, P., et al., 2018. Whole-body clearing, staining and screening of calcium deposits in the mdx mouse model of Duchenne muscular dystrophy. Skeletal Muscle 8, 1-12.

Candeo, A., Sana, I., Ferrari, E., et al., 2016. Virtual unfolding of light sheet fluorescence microscopy dataset for quantitative analysis of the mouse intestine. J. Biomed. Opt. 21, 056001.

Chakraborty, T., Driscoll, M.K., Jeffery, E., et al., 2019. Light-sheet microscopy of cleared tissues with isotropic, subcellular resolution. Nat. Methods 16, 1109-1113.

Chang, C.C., Chu, A., Meyer, S., et al., 2021. Three-dimensional imaging coupled with topological quantification uncovers retinal vascular plexuses undergoing obliteration. Theranostics 11, 1162-1175.

Chen, Y., Xie, W., Glaser, A.K., et al., 2019. Rapid pathology of lumpectomy margins with open-top light-sheet (OTLS) microscopy. Biomed. Opt Express 10, 1257.

Chung, K., Wallace, J., Kim, S.Y., et al., 2013. Structural and molecular interrogation of intact biological systems. Nature 497, 332-337.

Dean, K.M., Roudot, P., Welf, E.S., et al., 2015. Deconvolution-free subcellular imaging with axially swept light sheet microscopy. Biophys. J. 108, 2807-2815.

Dean, K.M., Roudot, P., Reis, C.R., et al., 2016. Diagonally scanned light-sheet microscopy for fast volumetric imaging of adherent cells. Biophys. J. 110, 1456-1465.

Di Giovanna, A.P., Tibo, A., Silvestri, L., et al., 2018. Whole-brain vasculature reconstruction at the single capillary level. Sci. Rep. 8, 1-11.

Ding, Y., Gudapati, V., Lin, R., et al., 2020. Saak transform-based machine learning for light-sheet imaging of cardiac trabeculation. IEEE Trans. Biomed. Eng. 9294. https://doi.org/10.1109/TBME.2020.2991754.

Dodt, H.U., Leischner, U., Schierloh, A., et al., 2007. Ultramicroscopy: three- dimensional visualization of neuronal networks in the whole mouse brain. Nat. Methods 4, 331-336.

d'Esposito, A., Sweeney, P.W., Ali, M., et al., 2018. Computational fluid dynamics with imaging of cleared tissue and of in vivo perfusion predicts drug uptake and treatment responses in tumours. Nat Biomed Eng 2, 773-787.

Ertürk, A., Mauch, C.P., Hellal, F., et al., 2012. Three-dimensional imaging of the unsectioned adult spinal cord to assess axon regeneration and glial responses after injury. Nat. Med. 18, 166-171.

Ertürk, A., Lafkas, D., Chalouni, C., 2014. Imaging cleared intact biological systems at a cellular level by 3DISCO. JoVE 1-10.

Frasconi, P., Silvestri, L., Soda, P., et al., 2014. Large-scale automated identification of mouse brain cells in confocal light sheet microscopy images. Bioinformatics 30, 587-593.

Fricker, M.D., 1993. Optical microscopy: emerging methods and applications. FEBS Lett. 335, 145-145.

Fuchs, E., Jaffe, J., Long, R., et al., 2002. Thin laser light sheet microscope for microbial oceanography. Opt Express 10, 145.

Gao, L., 2015. Extend the field of view of selective plane illumination microscopy by tiling the excitation light sheet. Opt Express 23, 6102.

Gebhardt, J.C.M., Suter, D.M., Roy, R., et al., 2013. Single-molecule imaging of transcription factor binding to DNA in live mammalian cells. Nat. Methods 10, $421-426$.

Girkin, J.M., Carvalho, M.T., 2018. The light-sheet microscopy revolution. J. Opt. 20 https://doi.org/10.1088/2040-8986/aab58a.

Glaser, A.K., Reder, N.P., Chen, Y., et al., 2017. Light-sheet microscopy for slide-free non-destructive pathology of large clinical specimens. Nat Biomed Eng 1. https://doi.org/10.1038/s41551-017-0084.

Glaser, A.K., Chen, Y., Yin, C., et al., 2018. Multidirectional digital scanned light-sheet microscopy enables uniform fluorescence excitation and contrast-enhanced imaging. Sci. Rep. 8, 2-12.

Gómez-Gaviro, M.V., Balaban, E., Bocancea, D., et al., 2017. Optimized CUBIC protocol for three-dimensional imaging of chicken embryos at single-cell resolution. Development 144, 2092-2097.

Gómez-Gaviro, M.V., Sanderson, D., Ripoll, J., et al., 2020. Biomedical applications of tissue clearing and three-dimensional imaging in health and disease. iScience 23. https://doi.org/10.1016/j.isci.2020.101432.

Guo, Y., Wang, Q., Krupa, O., et al., 2019. Cross modality microscopy segmentation via adversarial adaptation. In: Rojas, I., Valenzuela, O., Rojas, F., et al. (Eds.), Bioinformatics and Biomedical Engineering. Springer International Publishing, Cham, pp. 469-478.

Gustavsson, A.K., Petrov, P.N., Lee, M.Y., et al., 2018. 3D single-molecule super-resolution microscopy with a tilted light sheet. Nat. Commun. 9, 1-8.

Hägerling, R., Drees, D., Scherzinger, A., et al., 2017. VIPAR, a quantitative approach to 3D histopathology applied to lymphatic malformations. JCI Insight 2, 1-14.

Hahn, A., Bode, J., Schuhegger, S., et al., 2020. Brain tumor classification of virtual NMR voxels based on realistic blood vessel-induced spin dephasing using support vector machines. NMR Biomed. 1-17.

Hama, H., Kurokawa, H., Kawano, H., et al., 2011. Scale: a chemical approach for fluorescence imaging and reconstruction of transparent mouse brain. Nat. Neurosci. 14, 1481-1488.

Hong, S.M., Jung, D.J., Kiemen, A., et al., 2020. Three-dimensional visualization of cleared human pancreas cancer reveals that sustained epithelial-tomesenchymal transition is not required for venous invasion. Mod. Pathol. 33, 639-647.

Hu, C., Hui, H., Wang, S., et al., 2017a. Cerebral vessels segmentation for light-sheet microscopy image using convolutional neural networks. In: Medical Imaging 2017: Biomedical Applications in Molecular, Structural, and Functional Imaging, 101370K.

Hu, C., Liu, X., Liang, X., et al., 2017b. Brain vascular image segmentation based on fuzzy local information C-means clustering. In: Imaging, Manipulation, and Analysis of Biomolecules, Cells, and Tissues XV, 100680Q.

Huisken, J., Stainier, D.Y.R., 2007. Even fluorescence excitation by multidirectional selective plane illumination microscopy (mSPIM). Opt. Lett. 32, 2608.

Huisken, J., Swoger, J., Del Bene, F., et al., 2004. Optical sectioning deep inside live embryos by selective plane illumination microscopy. Science (80- ) 305, 1007-1009.

Isaacson, D., Shen, J., McCreedy, D., et al., 2017. Dichotomous branching of human fetal lung demonstrated with light sheet fluorescence microscopy. Am. J. Respir. Crit. Care Med. 196, 1476-1477.

Isaacson, D., Shen, J., McCreedy, D., et al., 2018. Lightsheet fluorescence microscopy of branching human fetal kidney. Kidney Int. 93, 389-525.

Jahr, W., Schmid, B., Schmied, C., et al., 2015. Hyperspectral light sheet microscopy. Nat. Commun. 6, 1-7.

Jährling, N., Becker, K., Kramer, E.R., et al., 2008. 3D-Visualization of nerve fiber bundles by ultramicroscopy. Med. Laser Appl. 23, 209-215.

Jährling, N., Becker, K., Dodt, H.-U., 2009. 3D-reconstruction of blood vessels by ultramicroscopy. Organogenesis 5, 227-230.

Järling, N., Becker, K., Schönbauer, C., et al., 2010. Three-dimensional reconstruction and segmentation of intact Drosophila by ultramicroscopy. Front. Syst. Neurosci. $4,1-6$.

Keller, P.J., Dodt, H.U., 2012. Light sheet microscopy of living or cleared specimens. Curr. Opin. Neurobiol. 22, 138-143.

Keller, P.J., Schmidt, A.D., Wittbrodt, J., et al., 2008. Reconstruction of zebrafish early embryonic development by scanned light sheet microscopy. Science (80- ) 322, 1065-1069. 
Kennel, P., Teyssedre, L., Colombelli, J., et al., 2018a. Toward quantitative threedimensional microvascular networks segmentation with multiview lightsheet fluorescence microscopy. J. Biomed. Opt. 23, 1.

Kennel, P., Teyssedre, L., Colombelli, J., et al., 2018b. Toward quantitative threedimensional microvascular networks segmentation with multiview lightsheet fluorescence microscopy. J. Biomed. Opt. 23, 1.

Kennel, P., Dichamp, J., Barreau, C., et al., 2020. From whole-organ imaging to insilico blood flow modeling: a new multi-scale network analysis for revisiting tissue functional anatomy. PLoS Comput. Biol. 16, e1007322.

Klingberg, A., Hasenberg, A., Ludwig-Portugall, I., et al., 2017. Fully automated evaluation of total glomerular number and capillary tuft size in nephritic kidneys using lightsheet microscopy. J. Am. Soc. Nephrol. 28, 452-459.

Köster, I., Haas, P., 2015. Light sheet microscopy turned vertically. Opt. Photonik 10, 39-43.

Leischner, U., Schierloh, A., Zieglgänsberger, W., et al., 2010. Formalin-induced fluorescence reveals cell shape and morphology in biological tissue samples. PloS One 5. https://doi.org/10.1371/journal.pone.0010391.

Lim, J., Lee, H.K., Yu, W., et al., 2014. Light sheet fluorescence microscopy (LSFM); past, present and future. Analyst 139 (19), 4758-4768.

Lin, H.-C.A., Dutta, R., Mandal, S., et al., 2017. Light-sheet microscopy for quantitative ovarian folliculometry. Diagnosis Treat Dis. Breast Reprod. Syst. 10043, 100430K.

Lloyd-Lewis, B., Davis, F.M., Harris, O.B., et al., 2016. Imaging the mammary gland and mammary tumours in 3D: optical tissue clearing and immunofluorescence methods. Breast Cancer Res. 18, 1-17.

Mano, T., Albanese, A., Dodt, H.-U., et al., 2018. Whole-brain analysis of cells and circuits by tissue clearing and light-sheet microscopy. J. Neurosci. 38, 9330-9337.

Mayer, J., Robert-Moreno, A., Danuser, R., et al., 2014. OPTiSPIM: integrating optical projection tomography in light sheet microscopy extends specimen characterization to nonfluorescent contrasts. Opt. Lett. 39, 1053.

Medeiros, G De, Norlin, N., Gunther, S., et al., 2015. Confocal multiview light-sheet microscopy. Nat. Commun. 6, 1-8.

Migliori, B., Datta, M.S., Dupre, C., et al., 2018. Light sheet theta microscopy for rapid high-resolution imaging of large biological samples. BMC Biol. 16, 1-19.

Morawski, M., Kirilina, E., Scherf, N., et al., 2018. Developing 3D microscopy with CLARITY on human brain tissue: towards a tool for informing and validating MRI-based histology. Neuroimage 182, 417-428.

Nehrhoff, I., Ripoll, J., Samaniego, R., et al., 2017. Looking inside the heart: a seethrough view of the vascular tree. Biomed. Opt Express 8, 3110.

Noë, M., Rezaee, N., Asrani, K., et al., 2018. Immunolabeling of cleared human pancreata provides insights into three-dimensional pancreatic anatomy and pathology. Am. J. Pathol. 188, 1530-1535.

Nojima, S., Susaki, E.A., Yoshida, K., et al., 2017. CUBIC pathology: three-dimensional imaging for pathological diagnosis. Sci. Rep. 7, 1-14.

Pan, C., Cai, R., Quacquarelli, F.P., et al., 2016. Shrinkage-mediated imaging of entire organs and organisms using uDISCO. Nat. Methods 13, 859-867.

Pan, C., Schoppe, O., Parra-Damas, A., et al., 2019. Deep learning reveals cancer metastasis and therapeutic antibody targeting in the entire body. Cell 179, 1661-1676.e19.

Pawley, J.B., 2006. Handbook of Biological Confocal Microscopy, third ed. Springer US. https://doi.org/10.1007/978-0-387-45524-2.

Perens, J., Salinas, C.G., Skytte, J.L., et al., 2020. An optimized mouse brain atlas for automated mapping and quantification of neuronal activity using iDISCO + and light sheet fluorescence microscopy. Neuroinformatics. https://doi.org/10.1007/ s12021-020-09490-8.

Perin, P., Voigt, F.F., Bethge, P., et al., 2019. iDISCO+ for the study of neuroimmune architecture of the rat auditory brainstem. Front. Neuroanat. 13, 1-5.

Pietzsch, T., Saalfeld, S., Preibisch, S., et al., 2015. BigDataViewer: visualization and processing for large image data sets. Nat. Methods 12, 481-483.

Pitrone, P.G., Schindelin, J., Stuyvenberg, L., et al., 2013. OpenSPIM: an open-access light-sheet microscopy platform. Nat. Methods 10, 598-599.

Reder, N.P., Glaser, A.K., McCarty, E.F., et al., 2019. Open-top light-sheet microscopy image atlas of prostate core needle biopsies. Arch. Pathol. Lab Med. 143, 1069-1075.

Renier, N., Wu, Z., Simon, D.J., et al., 2014. IDISCO: a simple, rapid method to immunolabel large tissue samples for volume imaging. Cell 159, 896-910.

Renier, N., Adams, E.L., Kirst, C., et al., 2016. Mapping of brain activity by automated volume Analysis of immediate early genes. Cell 165, 1789-1802.

Rocha, M.D., Düring, D.N., Bethge, P., et al., 2019. Tissue clearing and light sheet microscopy: imaging the unsectioned adult zebra finch brain at cellular resolution. Front. Neuroanat. 13, 1-7.

Ronneberger, O., Fischer, P., Brox, T., 2015. U-net: convolutional networks for biomedical image segmentation, 9351, 234-241.

Roostalu, U., Skytte, J.L., Salinas, C.G., et al., 2020. 3D quantification of changes in pancreatic islets in mouse models of diabetes type I and II. DMM Dis. Model Mech. 13. https://doi.org/10.1242/dmm.045351.
Saritas, T., Puelles, V.G., Su, X.T., et al., 2018. Optical clearing in the kidney reveals potassium-mediated tubule remodeling. Cell Rep. 25, 2668-2675.e3.

Scherzinger, A., Kleene, F., Dierkes, C., et al., 2016. Automated segmentation of immunostained cell nuclei in 3D ultramicroscopy images. Lect. Notes Comput. Sci. 9796, 105-116.

Sharpe, J., Ahlgren, U., Perry, P., et al., 2002. Optical projection tomography as a tool for 3D microscopy and gene expression studies. Science (80- ) 296, 541-545.

Silvestri, L., Bria, A., Sacconi, L., et al., 2012. Confocal light sheet microscopy: micronscale neuroanatomy of the entire mouse brain. Opt Express 20, 20582.

Silvestri, L., Paciscopi, M., Soda, P., et al., 2015. Quantitative neuroanatomy of all Purkinje cells with light sheet microscopy and high-throughput image analysis. Front. Neuroanat. 9, 1-11.

Sommer, C., Straehle, C., Kothe, U., et al., 2011. Ilastik: interactive learning and segmentation toolkit. Proc. - Int. Symp. Biomed. Imag. 230-233.

Spalteholtz, W., 1911. Ober das Durchsichtigmachen yon menschlichen und tierischen Prfiparaten und seine theoretischen Bedingungen. S. Hirzel, Leipzig.

Stefaniuk, M., Gualda, E.J., Pawlowska, M., et al., 2016. Light-sheet microscopy imaging of a whole cleared rat brain with Thy1-GFP transgene. Sci. Rep. 6, 1-9.

Strnad, P., Gunther, S., Reichmann, J., et al., 2016. Inverted light-sheet microscope for imaging mouse pre-implantation development. Nat. Methods 13, 139-142.

Susaki, E.A., Tainaka, K., Perrin, D., et al., 2015. Advanced CUBIC protocols for wholebrain and whole-body clearing and imaging. Nat. Protoc. 10, 1709-1727.

Syenina, A., Vijaykrishna, D., Gan, E.S., et al., 2020. Positive epistasis between viral polymerase and the $3^{\prime}$ untranslated region of its genome reveals the epidemiologic fitness of dengue virus. Proc. Natl. Acad. Sci. U. S. A. 117, 11038-11047.

Tanaka, N., Kanatani, S., Kaczynska, D., et al., 2017. Whole-tissue biopsy phenotyping of three-dimensional tumours reveals patterns of cancer heterogeneity. Nat Biomed Eng 1, 796-806.

Tanaka, N., Kaczynska, D., Kanatani, S., et al., 2018. Mapping of the threedimensional lymphatic microvasculature in bladder tumours using light-sheet microscopy. Br. J. Canc. 118, 995-999.

Thierbach, K., Bazin, P.L., de Back, W., et al., 2018. Combining deep learning and active contours opens the way to robust, automated analysis of brain cytoarchitectonics. Lect. Notes Comput. Sci. 11046, 179-187.

Tischer, C., Ravindran, A., Reither, S., et al., 2020. BigDataProcessor2: a free and open-source Fiji plugin for inspection and processing of TB sized image data. bioRxiv, 244095.

Tomer, R., Lovett-Barron, M., Kauvar, I., et al., 2015. SPED light sheet microscopy: fast mapping of biological system structure and function. Cell 163, 1796-1806.

Tosi S, Bardia L, Filgueira MJ, et al. LOBSTER: an Environment to Design Bioimage Analysis Workflows for Large and Complex Fluorescence Microscopy Data. doi: 10.1093/bioinformatics/btz945/5682411.

Tosi, S., Bardia, L., Barallobre, M.J., et al., 2020. MosaicExplorerJ: interactive stitching of terabyte-size tiled datasets from lightsheet microscopy. F1000Research 9, 1308.

Ueda, H.R., Ertürk, A., Chung, K., et al., 2020a. Tissue clearing and its applications in neuroscience. Nat. Rev. Neurosci. https://doi.org/10.1038/s41583-019-0250-1.

Ueda, H.R., Ertürk, A., Chung, K., et al., 2020b. Tissue clearing and its applications in neuroscience. Nat. Rev. Neurosci. https://doi.org/10.1038/s41583-019-0250-1.

Voie, A.H., Burns, D.H., Spelman, F.A., 1993. Orthogonal-plane fluorescence optical sectioning: three-dimensional imaging of macroscopic biological specimens. J. Microsc. 170, 229-236.

Voigt, F.F., Kirschenbaum, D., Platonova, E., et al., 2019. The mesoSPIM initiative: open-source light-sheet mesoscopes for imaging in cleared tissue. bioRxiv, 577122.

Voleti, V., Patel, K.B., Li, W., et al., 2019. Real-time volumetric microscopy of in vivo dynamics and large-scale samples with SCAPE 2.0. Nat. Methods 16, 1054-1062.

Von Diezmann, A., Shechtman, Y., Moerner, W.E., 2017. Three-dimensional localization of single molecules for super-resolution imaging and single-particle tracking. Chem. Rev. 117, 7244-7275.

Yang, B., Treweek, J.B., Kulkarni, R.P., et al., 2014. Single-cell phenotyping within transparent intact tissue through whole-body clearing. Cell 158, 945-958.

Yang, Z., Mei, L., Xia, F., et al., 2015. Dual-slit confocal light sheet microscopy for in vivo whole-brain imaging of zebrafish. Biomed. Opt Express 6, 1797.

Yang, L., Feuchtinger, A., Möller, W., et al., 2019. Three-dimensional quantitative Comapping of pulmonary morphology and nanoparticle distribution with cellular resolution in nondissected murine lungs. ACS Nano 13, 1029-1041.

Zagato, E., Brans, T., Verstuyft, S., et al., 2017. Microfabricated devices for single objective single plane illumination microscopy (SoSPIM). Opt Express 25, 1732.

Zhang, H., Fang, C., Xie, X., et al., 2019. High-throughput, high-resolution deep learning microscopy based on registration-free generative adversarial network. Biomed. Opt Express 10, 1044.

Zundler, S., Klingberg, A., Schillinger, D., et al., 2017. Three-dimensional crosssectional light-sheet microscopy imaging of the inflamed mouse gut. Gastroenterology 153, 898-900. 